\title{
HDAC6 involves in regulating the IncRNA- microRNA-mRNA network to promote the proliferation of glioblastoma cells
}

\author{
An-Chih Wu ${ }^{1}$, Wen-Bin Yang ${ }^{1,2,3 \dagger}{ }^{\dagger}$, Kwang-Yu Chang ${ }^{4 \dagger}$, Jung-Shun Lee ${ }^{5 \dagger}$, Jing-Ping Liou ${ }^{6 \dagger}$, Ruei-Yuan Su${ }^{1}$, \\ Siao Muk Cheng ${ }^{4}$, Daw-Yang Hwang ${ }^{4}$, Ushio Kikkawa' ${ }^{1}$, Tsung-I Hsu ${ }^{2,3,7,8}$, Chih-Yang Wang ${ }^{9}$, Wen-Chang Chang ${ }^{1}$, \\ Pin-Yuan Chen ${ }^{10^{*}}$ and Jian-Ying Chuang $2,3,7,8,11^{*}$ (D)
}

\begin{abstract}
Background: Glioblastoma (GBM) is the most aggressive and lethal brain tumor. Although the histone deacetylase (HDAC)/transcription factor axis promotes growth in GBM, whether HDACs including HDAC6 are involved in modulating long non-coding RNAs (IncRNAs) to affect GBM malignancy remains obscure.

Methods: Integrative analysis of microarray and RNA-seq was performed to identify IncRNAs governed by HDAC6. Half-life measurement and RNA-protein pull-down assay combined with isobaric tags for relative and absolute quantitation (iTRAQ)-based proteomic analysis were conducted to identify RNA modulators. The effect of LINC00461 on GBM malignancy was evaluated using animal models and cell proliferation-related assays. Functional analysis of the LINC00461 downstream networks was performed comprehensively using ingenuity pathway analysis and public databases.

Results: We identified a IncRNA, LINC00461, which was substantially increased in stem-like/treatment-resistant GBM cells. LINC00461 was inversely correlated with the survival of mice-bearing GBM and it was stabilized by the interaction between HDAC6 and RNA-binding proteins (RBPs) such as carbon catabolite repression-negative on TATA-less (CCR4-NOT) core exoribonuclease subunit 6 and fused in sarcoma. Targeting LINC00461 using azaindolylsulfonamide, an HDAC6 inhibitor, decreased cell-division-related proteins via the IncRNA-microRNA (miRNA)-mRNA networks and caused cell-cycle arrest, thereby suppressing proliferation in parental and drug-resistant GBM cells and prolonging the survival of mice-bearing GBM.
\end{abstract}

Conclusions: This study sheds light on the role of LINC00461 in GBM malignancy and provides a novel therapeutic strategy for targeting the HDAC6/RBP/LINC00461 axis and its downstream effectors in patients with GBM.

Keywords: Glioblastoma, Histone deacetylase 6, IncRNA LINC00461, RNA-binding proteins

*Correspondence: pinyuanc@cgmh.org.tw; chuangcy@tmu.edu.tw tWen-Bin Yang, Kwang-Yu Chang, Jung-Shun Lee and Jing-Ping Liou contributed equally to this work.

${ }^{2}$ The Ph.D. Program for Neural Regenerative Medicine, Taipei Medical University, 250 Wuxing Street, Taipei 11031, Taiwan

${ }^{10}$ Department of Neurosurgery, Keelung Chang Gung Memorial Hospital, Chang Gung University, 222 Mai-jin Road, Keelung 20401, Taiwan

Full list of author information is available at the end of the article

\section{Background}

Glioblastoma (GBM) has a poor prognosis and remains incurable, despite aggressive treatment options including surgery, radiation therapy, and first-line chemotherapy with the drug temozolomide (TMZ). Less than $5 \%$ of the patients with GBM survive for 5 years following diagnosis. Approximately $90 \%$ of patients suffer from disease relapse within 2 years of treatment, regardless of their original author(s) and the source, provide a link to the Creative Commons licence, and indicate if changes were made. The images or other third party material in this article are included in the article's Creative Commons licence, unless indicated otherwise in a credit line to the material. If material is not included in the article's Creative Commons licence and your intended use is not permitted by statutory regulation or exceeds the permitted use, you will need to obtain permission directly from the copyright holder. To view a copy of this licence, visit http://creativecommons.org/licenses/by/4.0/. The Creative Commons Public Domain Dedication waiver (http://creativeco mmons.org/publicdomain/zero/1.0/) applies to the data made available in this article, unless otherwise stated in a credit line to the data. 
initial response to prior therapy [1]. Previous studies have revealed that GBM frequently harbors different gene expression patterns, causing patients to exhibit diverse clinical characteristics, survival times, and responses to treatment $[2,3]$. Therefore, understanding the molecular mechanisms of GBM and developing novel strategies for treating the disease are urgent.

Although GBM is characterized by chromosome 10q loss, aberrant expression of proteins, such as epidermal growth factor receptor amplification and $p 16^{I N K 4 a}$ deletion, and gene mutations, such as TP53, IDH1, and PTEN, the molecular basis of the onset and progression of GBM malignancy is not fully understood $[4,5]$. Because protein-coding genes account for $<2 \%$ of the whole genome, examining the impact of non-coding genes on the regulation of the glioma phenotype appears essential [6]. Long non-coding RNAs (lncRNAs) are defined as transcripts exceeding 200 base pairs in length; these molecules have a wide variety of biological functions [7]. Accumulating evidence has revealed that many dysregulated lncRNAs contribute to chemoresistance and poor prognostic features of GBM [8]. As a result, identification of lncRNA as a potential target for treating patients with GBM is urgently needed.

The structure of lncRNA is comparable to that of mRNA, with a $7 \mathrm{mGpppG} 5^{\prime}$ cap and $3^{\prime}$-polyadenosine tail. Similar to mRNA decay, lncRNA stabilization and degradation are regulated by microRNAs, decay-promoting RNA-binding proteins (RBPs), decapping enzymes, and deadenylases [9]. Our previous study has demonstrated that azaindolylsulfonamide (MPT0B291), a histone deacetylase (HDAC) inhibitor selective for HDAC6 $\left(\mathrm{IC}_{50} \approx 0.0052 \mu \mathrm{M}\right)[10,11]$, induces cellular senescence in stem-like GBM cells and prolongs the survival of mice-bearing temozolomide (TMZ)-resistant xenografts through downregulation of $\mathrm{Sp} 1$ and its targeted genes associated with drug resistance [10]. Although HDAC6 is known to maintain the acetylation balance of histones and nonhistone substrates, such as $\alpha$-tubulin, cortactin, HSP90, and a few transcriptional factors [12], whether it participates in the regulation of lncRNAs that affect tumorigenesis remains obscure.

In the current study, we used integrative microarray and RNA-seq analysis to investigate whether HDAC6 modulates lncRNAs. A highly conserved lncRNA, LINC00461, which functions as an essential regulator in glioma formation [13] and regulates the expressions of genes such as DNA topoisomerase II Alpha (TOP2A) [13], B-cell lymphoma 2 (BCL2) [14], and integrin $\beta 3$ (ITGB3) [15], was identified to be remarkably downregulated by HDAC6 depletion. Our data highlight LINC00461-associated regulatory networks in GBM malignancies, including treatment resistance and cancer stemness, and provide a novel insight into targeting the HDAC6/RBP/LINC00461 axis as a therapeutic approach for patients with GBM.

\section{Materials and methods \\ Materials}

MPT0B291 [11], synthesized by Prof. J. P Liou at Taipei Medical University, was dissolved in dimethyl sulfoxide (DMSO; Sigma-Aldrich Corp., St. Louis, MO, USA). Temozolomide (TMZ) and trichostatin A (TSA) were purchased from MedChemExpress (Monmouth Junction, NJ, USA) and Santa Cruz Biotechnology, Inc. (Dallas, TX, USA), respectively.

\section{Cell culture}

Human GBM cell lines, including U87MG (ATCC HTB14; American Type Culture Collection, Manassas, VA, USA) and A172 (ATCC CRL-1620), and patient-derived primary GBM (Pt\#3 and Pt\#5) along with their TMZresistant and stem-like cells, were cultured in respective media as described previously [16]. Procedures utilized for establishing TMZ-resistant GBM cells were mentioned previously [16]. To maintain TMZ-resistant cells, $50 \mu \mathrm{M}$ TMZ was added into the culture medium, and their resistance characteristics were confirmed using colony-formation assay. Informed consent obtained from the patients followed the protocols (Nos. 201,006,011 and $201,402,018$ ) approved by the Joint Institutional Review Board (JIRB) of Taipei Medical University (Taipei, Taiwan).

\section{Transient transfection}

FLAG-tagged CNOT6 (Sino Biological, Inc., Beijing, China) or the empty vector with FLAG tag was transfected into U87MG cells using PolyJet (SignaGen Laboratories, Frederick, MD, USA).

\section{Stable cell line construction}

The expression vector pcDNA3.1(-) carrying LINC00461 inserts (MDBio, Taipei, Taiwan) was transformed into Escherichia coli directly, and the DNA extracted from ampicillin (Sigma-Aldrich Corp.)-resistant bacteria was amplified using PCR. Pt\#3 cells transiently transfected with pcDNA3.1-LINC00461 using PolyJet (SignaGen Laboratories). Two days after transfection, cells stably expressing pcDNA3.1-LINC00461 were selected by treating with $0.5 \mathrm{mg} / \mathrm{mL}$ geneticin (G418; Thermo Fisher Scientific, Waltham, MA, USA).

\section{RNA interference}

Parental and TMZ-resistant GBM cells (U87MG, $\mathrm{A} 172$, and Pt\#3) were transfected with 5,10 , or $20 \mathrm{nM}$ specific gene siRNA or control non-targeting siRNA 
(ON-TARGETplus siRNA-SMARTpool/non-targeting pool, Dharmacon, Inc., Lafayette, CO, USA) using lipofectamine 3000 (Thermo Fisher Scientific). Seventy-two hours after the transfection of siRNA, the total RNA was extracted from GBM cells using TRIzol reagent (Thermo Fisher Scientific). The effects of depletion were validated by RT-qPCR. Targeted sequences for each gene are listed in Table S1.

\section{Microarray analysis}

Stem-like and TMZ-resistant U87MG cells were treated with 10 and $6 \mu \mathrm{M}$ MPT0B291, respectively, for 1 day. U87MG cells were treated with si-Sp1 for 3 days. Gene expression analysis following total RNA extracted from these cells using TRIzol reagent (Thermo Fisher Scientific) was performed by Welgene Biotech (Taipei, Taiwan) using a SurePrint G3 Human Gene Expression 8x60K Microarray (Agilent Technologies, Inc., Santa Clara, CA, USA). The number of non-coding genes with the signalto-noise-ratio (SNR) threshold of $>1.0$ was identified.

\section{RNA-seq}

RNA-seq was performed by Welgene Biotech. In brief, after HDAC6 or LINC00461 depletion, respectively, in TMZ-resistant U87MG and parental U87MG cells, the total RNA was extracted using Trizol Reagent (Thermo Fisher Scientific). Purified RNA was quantified at OD260 $\mathrm{nm}$ using an ND-1000 spectrophotometer (NanoDrop Technologies, Inc. Wilmington, DE, USA) and qualified using a Bioanalyzer 2100 (Agilent Technologies, Inc.) with RNA 6000 LabChip kit (Agilent Technologies, Inc.). The RNA-Seq libraries were constructed using Agilent's SureSelect Strand-Specific RNA Library Preparation Kit, followed by AMPure XP Beads (Beckman Coulter, Brea, CA, USA) size selection, and the sequence was directly determined using Illumina's sequencing-by-synthesis technology.

\section{Quantitative reverse transcription PCR (RT-qPCR)}

Total RNA was extracted from cells using TRIzol reagent (Thermo Fisher Scientific), followed by reverse transcription using SuperScript IV First-Strand Synthesis System (Thermo Fisher Scientific) and $\mathrm{TaqMan}^{\mathrm{TM}}$ MicroRNA Reverse Transcription Kit (Thermo Fisher Scientific), respectively, for lncRNA or mRNA and miRNA. qPCR analysis was performed using iTaq Universal SYBR Green Supermix (Bio-Rad Laboratories, Inc., Hercules, CA, USA) and TaqMan ${ }^{\mathrm{TM}}$ Small RNA Assays, respectively, for lncRNA or mRNA and miRNA on StepOnePlus RealTime PCR System (Applied Biosystems Inc., Waltham, MA, USA). Relative expression levels of target genes were determined using the delta-delta $\mathrm{Ct}\left(2^{-\Delta \Delta \mathrm{Ct}}\right)$ method and normalized against the glyceraldehyde 3-phosphate dehydrogenase (GAPDH) mRNA and U6 snRNA levels, respectively, for lncRNA or mRNA and miRNA. Oligonucleotides used for TaqMan qPCR assays were purchased from Suu-Flower Co., Ltd. (Taichung City, Taiwan). The primer or probe sequences for each gene are listed in Table S2.

\section{Animal models}

For the subcutaneous tumor model, $1 \times 10^{6} \mathrm{GBM}$ cells (U87MG and Pt\#3) were implanted into both dorsal flanks of female nonobese diabetic/severe combined immunodeficiency (NOD/SCID) mice (8weeks old, BioLASCO Co., Ltd., Taipei, Taiwan). Intraperitoneal administration with MPT0B291 $(10 \mathrm{mg} / \mathrm{kg})$ or vehicle (DMSO) every other day was initiated when the tumor volume reached a size of $10-20 \mathrm{~mm}^{3}$ after tumor implantation. Tumor volumes were measured using a caliper three times a week and calculated following the modified ellipsoidal formula: tumor volume $=1 / 2$ (length $\times$ width $^{2}$ ) [17]. Tumor weights were recorded at the end of the experiments. For the orthotopic tumor model, $5 \times 10^{5}$ Pt\#3 cells (with or without stable LINC00461 expression) were injected into the right frontal brain area of male Bagg albino (BALB)/c nude mice (9 weeks old, BioLASCO Co., Ltd.) according to detailed procedures reported previously [18]. Intraperitoneal administration with MPT0B291 $(10 \mathrm{mg} / \mathrm{kg})$ or vehicle (DMSO) every other day was initiated 5 days after tumor inoculation. Mice weights were recorded three times a week. All protocols for animal experiments were approved by the Institutional Animal Care and Use Committee of the National Health Research Institute (IACUC, NHRI, Tainan, Taiwan). Animal experiments were conducted under the registration number (NHRI-IACUC-106010).

\section{RNA-in situ hybridization (ISH) and RNA-fluorescence in situ hybridization (FISH)}

Digoxigenin (DIG)-labeled probes (Custom LNATM Detection Probes; Table S3) targeting LINC00461 or scramble were purchased from Qiagen (Hilden, Germany). In situ hybridization (ISH) was performed following the manufacturer's instructions with slight modifications from IsHyb in situ hybridization kit (BioChain Institute Inc., Newark, CA, USA). In brief, $5-\mu \mathrm{m}$ serial sections of MPT0B291-treated or control xenograft tumors were hybridized with DIG-labeled LINC00461 or scramble probes $(2-4 \mathrm{ng} / \mu \mathrm{L})$ at $55^{\circ} \mathrm{C}$ for $16 \mathrm{~h}$. For ISH, chromogenic reaction used alkaline phosphatase (AP)conjugated anti-DIG antibody (1:100) and 5-bromo4-chloro-3-indolyl phosphate/nitro blue tetrazolium provided by manufacturer to detect the expression. For FISH, rhodamine tetramethylrhodamine-isothiocyanate-conjugated anti-DIG (111-025-003, 1:100, Jackson 
ImmunoResearch Laboratories, Inc., West Grove, PA, USA) was used to detect the expression instead. Cell nuclei were counterstained with nuclear fast red (Vector Laboratories, Inc., Burlingame, CA, USA) and 4',6-diamidino-2-phenylindole (DAPI) (glycerol mounting medium - anti-fade with DAPI; ab188804, Abcam, Inc., Cambridge, UK), respectively, in ISH and FISH staining. Images of FISH staining were captured using ImageXpress Pico Automated Cell Imaging System (Molecular Devices, San Jose, CA, USA).

\section{Immunohistochemistry}

After antigen retrieval, immunohistochemistry was performed following the manufacturer's instructions with slight modifications using VECTASTAIN ${ }^{\circledR}$ ABC AP Kits (Vector Laboratories, Inc.). In brief, $5-\mu \mathrm{m}$ serial sections of MPT0B291-treated or control xenograft tumors were hybridized with an antibody against either Ki-67 (ab16667, 1:100, Abcam, Inc.) or cleaved caspase-3 (\#9661, 1:100, Cell Signaling Technology, Inc., Danvers, MA, USA). Average 3,3'-diaminobenzidine staining intensities of each slide normalized by the nuclei number were calculated using the semi-quantitative method [19].

\section{mRNA stability measurement}

Twenty-four hours after treating with MPT0B291 or vehicle control (DMSO), $10 \mu \mathrm{g} / \mathrm{mL}$ actinomycin D (ActD; Selleckchem, Houston, TX, USA) was added to inhibit transcription in TMZ-resistant Pt\#3 and Pt\#5 cells. RTqPCR was used to detect the half-life of LINC00461 at 0, $20,40,60$, and $120 \mathrm{~min}$ after transcriptional inhibition.

\section{Immunoprecipitation assay}

The cell lysate was prepared using radioimmunoprecipitation assay buffer containing protease inhibitor (Roche, Basel, Switzerland) and then immunoprecipitated with $2 \mu \mathrm{g}$ anti-HDAC6 (\#7612, Cell Signaling Technology, Inc.) or IgG (sc-2025/2027, Santa Cruz Biotechnology, Inc.) at $4{ }^{\circ} \mathrm{C}$ overnight, followed by incubation with protein A agarose (Merck Millipore, Bedford, MA, USA) at $4{ }^{\circ} \mathrm{C}$ for $1 \mathrm{~h}$. For the immunoprecipitation of FLAG-tagged CNOT6, anti-FLAG M2 affinity gel (Sigma-Aldrich Corp.) was used following the manufacturer's instructions. The immune complexes were detected by Western blot analyses with anti-CNOT6 (sc-81,231, 1:1000, Santa Cruz Biotechnology, Inc.), anti-HDAC6 (\#7612, 1:1000, Cell Signaling Technology, Inc.), and anti-acetyl-lysine (GTX80693, 1:1000, GeneTex Inc., Irvine, CA, USA) antibodies.

\section{RNA-protein pull-down assay}

Circular plasmid, pcDNA3.1-LINC00461, was converted to a linear template using EcoRV-HF digestion (New
England Biolabs Inc., Ipswich, MA, USA) for in vitro LINC00461 synthesis (HiScribe ${ }^{\mathrm{TM}}$ T7 Quick High Yield RNA Synthesis Kit; New England Biolabs). The pulldown of RNA-protein complexes was conducted using Pierce ${ }^{\mathrm{TM}}$ Magnetic RNA-Protein Pull-Down Kit (Thermo Fisher Scientific) based on the manufacturer's instructions. Briefly, $50 \mathrm{pmol}$ desthiobiotin-labeled (Pierce ${ }^{\mathrm{TM}}$ RNA $3^{\prime}$ End Biotinylation Kit, Thermo Fisher Scientific) androgen receptor (AR) 3'-untranslated regions (UTR) and LINC00461 were captured by $50 \mu \mathrm{L}$ streptavidin magnetic beads and then interacted with $200 \mu \mathrm{g}$ lysate extracted from parental and TMZ-resistant Pt\#3 cells. Elution of RNA-binding protein complexes was applied for western blotting and isobaric tags for relative and absolute quantitation (iTRAQ)-based proteomic analysis (Biotools Co. Ltd., New Taipei City, Taiwan). 3'-UTR of AR RNA, which contains UC-rich regions for human antigen $\mathrm{R}$ binding, is the control system for the pulldown assay.

\section{Cell proliferation assay}

Parental and TMZ-resistant cells (U87MG and A172) at a density of $10^{4}$ cells per well were seeded in 24-well tissue culture plates overnight. After downregulation of LINC00461 using siRNA, cell proliferation was determined by adding $0.5 \mathrm{mg} / \mathrm{mL} 3$-(4,5-dimethylthiazol-2-yl)2,5-diphenyltetrazolium bromide (MTT, BIONOVAS Biotechnology Co., Ltd., Toronto, CA) for $30 \mathrm{~min}$ at $37^{\circ} \mathrm{C}$. After the reaction, the formazan products were dissolved in $0.3 \mathrm{~mL}$ DMSO (Sigma-Aldrich Corp.), and the absorbance was spectrophotometrically measured at $550 \mathrm{~nm}$ every $24 \mathrm{~h}$ by an ELIZA reader (Bio-Rad Laboratories, Inc.).

\section{Cell-cycle analysis}

The cell cycle was evaluated by flow cytometry. Briefly, parental and TMZ-resistant U87MG cells at a density of $7.5 \times 10^{4}$ cells per well were seeded in 6 -cm petri dishes. After downregulation of LINC00461 using siRNA, cells were collected and fixed with $70 \%$ ethanol at $4{ }^{\circ} \mathrm{C}$ overnight. Fixed cells were incubated with $0.5 \mathrm{~mL}$ PBS containing $0.1 \%$ Triton X-100 (Pharmacia \& Upjohn Company LLC, North Peapack, NJ, USA) on ice for $15 \mathrm{~min}$ and then stained with propidium iodide (SigmaAldrich Corp.)/RNase A (BIONOVAS Biotechnology Co.) solution $(20 \mu \mathrm{g} / \mathrm{mL}$ propidium iodide and $0.3 \mathrm{mg} /$ $\mathrm{mL}$ RNase A in PBS) for $30 \mathrm{~min}$ in darkness. Stained cells were measured by flow cytometry (Guava easyCyte, Merck Millipore). Cell-cycle distribution was analyzed by calculating percentages of cells at subG1, G0/G1, S, and G2/M phases. 


\section{Western blot analysis}

For western blot analysis, anti-Sp1 (\#07-645, 1:1000, MilliporeSigma, Burlington, MA, USA), anti-HDAC1 (H3284, 1:1000, MilliporeSigma), anti-HDAC2 (\#05-814, 1:1000, MilliporeSigma), anti-HDAC6 (\#7612, 1:1000, Cell Signaling Technology, Inc.), anti-CNOT6 (sc-81,231, 1:1000, Santa Cruz Biotechnology, Inc.), anti-fused in sarcoma (FUS) (A5921, 1:1000, ABclonal Inc., Woburn, MA, USA), anti-Acetyl-lysine (GTX80693, 1:1000, GeneTex Inc.), anti-PABP (ab21060, 1:1000, Abcam, Inc.), antiTOP2A (MAB4197, 1:1000, MilliporeSigma), anti-Ki67 (A11005, 1:1000, ABclonal Inc.), anti-MELK (A3530, 1:1000, ABclonal Inc.), anti-DLGAP5 (A2197, 1:1000, ABclonal Inc.), anti-CD168 (A11666, 1:1000, ABclonal Inc.), anti-MCM10 (A5427, 1:1000, ABclonal Inc.), antiTMPO (A2534, 1:1000, ABclonal Inc.), anti-cyclin D1 (E1A6234, 1:1000, EnoGene Biotech Co., Ltd., New York, NY, USA), anti-CDK1 (sc-8395, 1:1000, Santa Cruz Biotechnology, Inc.), and anti-alpha Tubulin (66031-1-Ig, 1:10,000, Proteintech Group, Inc., Rosemont, IL, USA) were used as primary antibodies. HRP-linked anti-mouse IgG (sc-2004, 1:5000, Santa Cruz Biotechnology, Inc.) and HRP-linked anti-rabbit IgG (sc-2005, 1:5000, Santa Cruz Biotechnology, Inc.) were used as secondary antibodies. The signals were amplified using an enhanced chemiluminescence reagent (ECL, GE Healthcare, Chicago, IL, USA) and captured using the ChemiDoc Touch Imaging System (Bio-Rad Laboratories, Inc.). The intensity of each band was determined by Image Lab, and the relative target protein levels were normalized against the tubulin level.

\section{Single-cell RNA sequencing (scRNA-seq)}

Fresh tumor tissues from four patients with GBM were prepared for scRNA-seq following the protocol established by 10x Genomics ${ }^{\circledR}$ Single Cell (Pleasanton, CA, USA). Data analysis was performed using Cell Ranger $\left(10 x\right.$ Genomics $\left.^{\circledR}\right)$ and Seurat (29608179). Informed consent obtained from the patients followed a protocol (IRB No. EC1080202) approved by the Research Ethics Committee of the National Health Research Institute (NHRI, Miaoli, Taiwan).

\section{Analysis of databases}

Next-generation sequencing (NGS) databases of human GBM transcriptome obtained from The Cancer Genome Atlas (TCGA) (https://portal.gdc.cancer.gov) were collected and processed as described previously [10]. The expression fold change and significance level ( $t$-test) of 50 cell-division-related genes, which appeared in the intersection dataset of LINC00461 depletion and MPT0B291 treatment, between normal and GBM NGS data were calculated. The correlation of gene expression status between TCGA-GBM and LINC00461-silenced datasets was computed using Pearson's correlation coefficient (PCC). Prognostic implications of cell-division-related genes in high-grade glioma patients were analyzed using PROGgeneV2 (http://www.progtools.net/gene/) [20]. Three public databases, miRDB (http://mirdb.org/), miRWALK (http://mirwalk.umm.uni-heidelberg.de/), and starBase (http://starbase.sysu.edu.cn/index.php) were used to predict the lncRNA-miRNA-mRNA networks.

\section{Bioinformatic analyses}

The heatmap, which displayed the $\log 2$ (fold change) values of selected lncRNAs from microarray datasets, was generated using online software Morpheus (https://softw are.broadinstitute.org/morpheus). Functional analysis of data from microarray and RNA sequencing was performed using ingenuity pathway analysis (IPA, Qiagen). The overlap between molecules in our datasets and a particular function was calculated using the right-tailed Fisher's exact test. Prediction of activation or inhibition of pathways was examined using a Z-score. The heat map was generated using a MultiExperiment Viewer by the $\log 2$ (fold change) values of selected genes.

\section{Statistics}

The statistical significance of the difference between the two groups was analyzed using the unpaired Student's $t$-test with a two-tailed $p$-value. For more than two groups, one-way or two-way analysis of variance (ANOVA) was used to examine statistically significant differences depending on the number of variables. The survival curves of different groups were compared using the log-rank test. A $p$-value of $<0.05$ was considered statistically significant. The statistical significance increased in the order: $" p<0.05, " p<0.01,{ }^{* * *} p<0.001$, and ${ }^{* * a *} p<0.0001$.

\section{Results \\ HDAC6 inhibition significantly decreases LINC00461 expression}

To determine whether HDAC6 affects lncRNA regulation, we analyzed the expression levels of 9445 lncRNAs using microarray. Microarray gene expression data were applied independently on mRNA samples isolated from GBM stem-like cells (serum-free/ suspension-adapted tumorspheres) [21] and TMZresistant cells [18]. A comparison of MPT0B291treated with DMSO-treated conditions showed that 54 lncRNA probe sets represent reliable microarray signals (signal-to-noise ratio $\geq 1$, Fig. 1A). Among these, only two lncRNAs (LINC00461 and LINC01559) were commonly and significantly altered in stem-like (S-U87MG) and TMZ-resistant (R-U87MG) GBM 
A
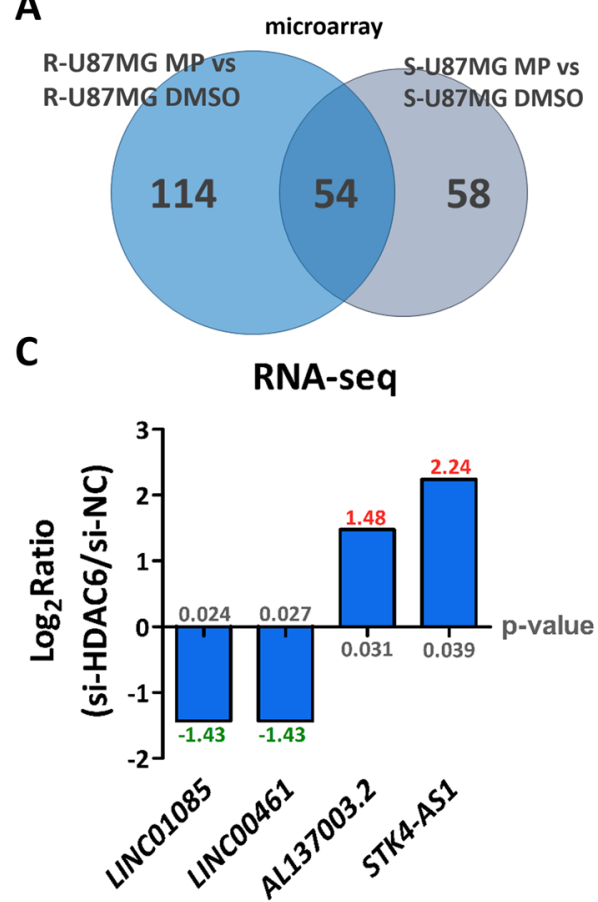

D
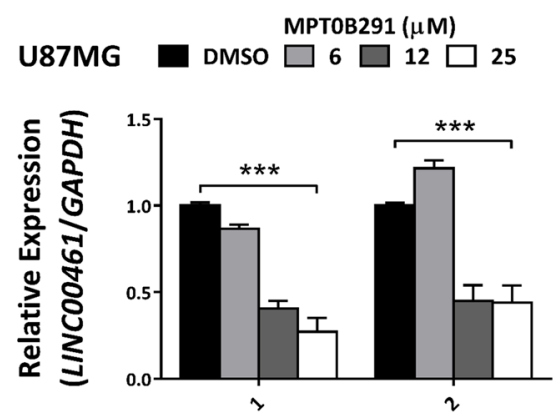

Days Post Treatment
B
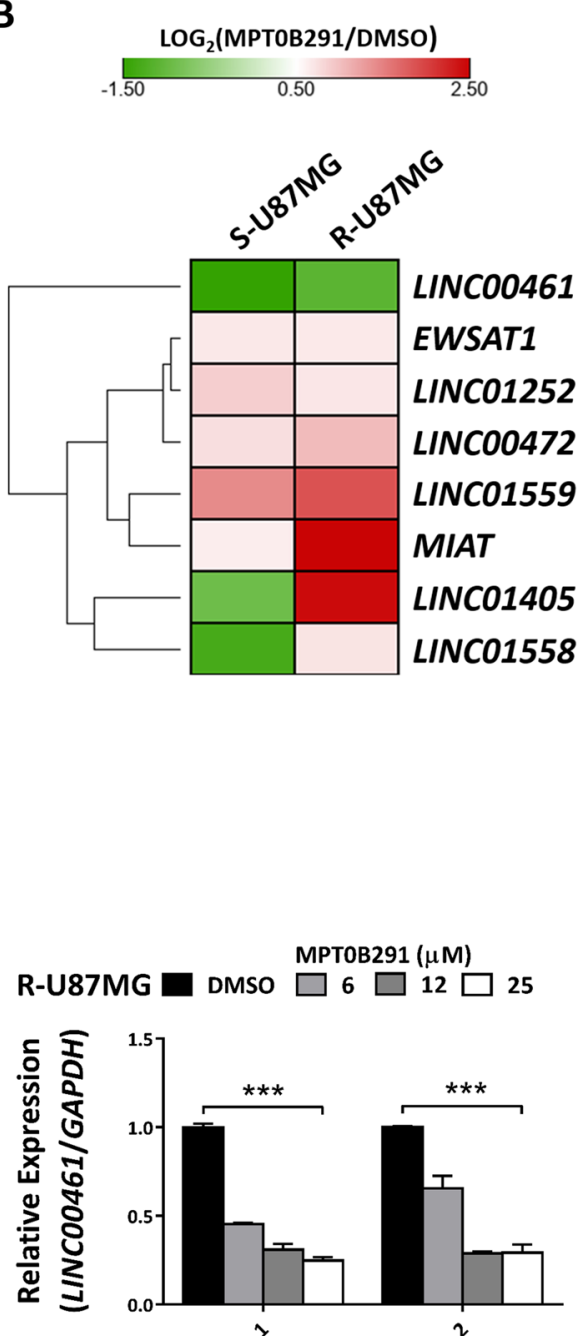

Days Post Treatment

Fig. 1 Significant reduction of LINC00461 expression in response to MPTOB291 treatment in glioblastomas (GBMs). A Venn diagram depicts the number of differentially expressed IncRNAs selected by microarray analysis in stem-like (S-U87MG) and temozolomide (TMZ)-resistant (R-U87MG) GBM cells treated with MPT0B291 (MP, $10 \mu$ M in S-U87MG; 6 mM in R-U87MG) and DMSO, respectively, for 1 day. B Heatmap represents the significantly changed expression of long non-coding RNAs (IncRNAs; fold change $\geq 1.5$ ) by MPTOB291 in the two microarray datasets in (A). The color scale indicates the relative fold change (log2) to control (DMSO) of each IncRNA, where red represents high expression and green represents low expression. Genes are hierarchically clustered based on their expression values. C RNA-seq revealed the discriminative expression of IncRNAs post HDAC6 knockdown. Y-axis represents relative expression level (log2) to control (si-NC) of each IncRNA shown in the x-axis. D Effect of MPTOB291 on LINC00461 expression in parental and TMZ-resistant U87MG cells after 1-2 days of treatment. The results are shown as mean \pm standard error of the mean (SEM) for triplicate samples in each group. One-way ANOVA

cells following MPT0B291 treatment (Fig. 1B, Fig. S1). LINC00461 levels were decreased 0.27 -fold and 0.49fold in S- and R-U87MG cells, respectively, while LINC01559 levels were upregulated 2.70-fold and 3.64fold in S- and R-U87MG cells, respectively. To further confirm the effect of HDAC6 on IncRNA regulation, we depleted HDAC6 using siRNA and performed RNA-seq analysis. Four lncRNAs showing altered levels after HDAC6 knockdown were identified (Fig. 1C).
Interestingly, a comparison of datasets obtained from MPT0B291 treatment and HDAC6 knockdown revealed that LINC00461 was the only lncRNA with an obvious reduction after HDAC6 inhibition, suggesting that it is a downstream IncRNA target of HDAC6. Moreover, further confirmation using RT-qPCR analysis showed a significant reduction in LINC00461 levels by MPT0B291 treatment in both parental and TMZresistant U87MG cells (Fig. 1D). 


\section{Attenuation of LINC00461 by MPTOB291 remarkably extends the survival time of mice with GBM}

Using U87MG xenografts and Pt\#3 xenografts in mice, we observed a significant reduction in tumor growth after MPT0B291 treatment (Fig. 2A-C). Moreover, we analyzed the expression of LINC00461 in MPT0B291treated and control xenografts by RNA ISH and RNA FISH. A decrease of approximately 40\% in LINC00461 molecules was visualized within the cytoplasm of MPT0B291-treated xenograft tissues compared to that in control tumors (Fig. 2D-E), confirming the suppressive effect of MPT0B291 on LINC00461 expression in vivo. Furthermore, MPT0B291 treatment extended the survival of mice-bearing intracranial GBM (Fig. 2F). To understand the mechanism by which MPT0B291 treatment prolonged survival through downregulating LINC00461, protein expressions of proliferation (Ki-67) and apoptosis (cleaved caspase-3) marker were measured in xenograft tissues. A significant reduction in Ki-67 expression was observed within the nucleus of MPT0B291-treated xenograft tissues compared to that in control tumors (Fig. S2A). In contrast, the difference in cleaved caspase-3 expression was not significant in the nucleus between MPT0B291-treated and control xenograft tissues (Fig. S2A), suggesting that the extension of survival in mice-bearing GBM is mainly derived from the anti-proliferative effect of MPT0B291. We then evaluated the role of LINC00461 on survival outcomes using the same orthotopic mouse model. Pt\#3 cells that stably express LINC00461 were constructed (Fig. 2G) and injected into the right cerebral hemisphere of mice. The LINC00461 high expression group's median survival was $24 \mathrm{~d}$, while that of the LINC00461 low expression group was 54 d (Fig. 2H). Thus, LINC00461 plays an important role in GBM growth, but HDAC6 inhibition induced by MPT0B291 blocks the oncogenic effect of LINC00461.

\section{HDAC6 and RNA-binding proteins are involved in managing LINC00461 expression}

MPT0B291 is known to block the activation of the HDAC6/Sp1 axis [10]. Whether MPT0B291 suppresses LINC00461 expression by a Sp1-dependent mechanism was further investigated. The result showed that LINC00461 expression was substantially decreased by MPT0B291 treatment while it increased with Sp1 depletion (Fig. 3A, bars 1-3). Consistently, RT-qPCR detection after Sp1 knockdown confirmed elevated LINC00461 levels in parental and TMZ-resistant U87MG cells (Fig. 3B), suggesting that MPT0B291-mediated downregulation of LINC00461 is modulated via an Sp1-independent pathway. Furthermore, to explore the mechanism underlying the modulation of LINC00461 expression, we investigated the RNA stability of LINC00461 using dactinomycin to block de novo RNA transcription in cells and found that MPT0B291 treatment caused 2.75-fold and 1.75-fold reduction in the half-life of LINC00461 in TMZ-resistant Pt\#3 and Pt\#5 cells, respectively (Fig. 3C). Considering that MPT0B291 is a selective inhibitor of HDAC6 and based on HDAC6 knockdown reduction of LINC00461 levels as verified by RNA-seq analysis (Fig. 1C) and RT-qPCR detection (Fig. 3D), HDAC6 was presumed to contribute to the regulation of LINC00461 stability. Additionally, we found that LINC00461 expression was higher in TMZ-resistant GBM cells. Increased expression of LINC00461 may correspond with the malignant behavior of GBM cells.

HDACs are known to regulate post-transcriptional gene expression by altering deadenylase complex activation and affecting polyadenylate tail stability of RNA [22]. Thus, we examined HDAC6 interaction with the catalytic subunits of the deadenylase complex and identified that carbon catabolite repression-negative on TATA-less (CCR4-NOT) core exoribonuclease subunit 6 (CNOT6), a typical protein related to mRNA decay, is associated with HDAC6 in U87MG cells (Fig. 3F). Furthermore, treating cells with HDAC6 inhibitors, MP0B291

\footnotetext{
(See figure on next page.)

Fig. 2 Suppression of LINC00461 by MPTOB291 inhibits tumor growth and improves survival of mice-bearing intracranial GBM. A Growth curves show average tumor volumes from $1 \times 10^{6} \mathrm{Pt}$ t\# cells and $1 \times 10^{6} \mathrm{U} 87 \mathrm{MG}$ cells injected into the dorsal flanks of NOD/SCID mice. MPTOB291 was administered after the tumor volume reached a size of $10-20 \mathrm{~mm}^{3}$ every other day ( $n \geq 5$ per group). Two-way ANOVA. B Average final tumor weights of control and MPTOB291-treated xenografts ( $n \geq 5$ per group). Unpaired Student's $t$-test. C Images of control and MPTOB291-treated xenografts at the end of the experiment ( $\mathrm{n} \geq 5$ per group). $\mathbf{D}$ ISH and FISH detected the expression of LINCO0461. Serial sections of control and MPTOB291-treated xenografts were hybridized with either a LINC00461 probe or a scramble probe in ISH and FISH, and cell nuclei were stained with nuclear fast red and DAPI, respectively, in ISH (left 4 panels) and FISH (right 4 panels) staining. Scale bars indicate $10 \mu \mathrm{m}$ (inset of ISH), $100 \mu \mathrm{m}$ (ISH), $13.07 \mu \mathrm{m}$ (inset of FISH), and $26.22 \mu \mathrm{m}$ (FISH). E Relative percentages of LINC00461 expression in the cells of control and MPTOB291-treated xenografts were measured by fluorescent intensity. F The Kaplan-Meier plot represents the survival time of BALB/C nude mice-bearing orthotopically implanted $5 \times 10^{5}$ Pt\#3 cells. MPTOB291 $(10 \mathrm{mg} / \mathrm{kg})$ was administered $5 \mathrm{~d}$ after cell implantation every other day ( $n=6$ per group). Log-rank test. G The expression levels of LINC00461 in Pt\#3 cells stably expressing either LINC00461 or empty vector control (pCDNA3.1). Unpaired Student's t-test. $\mathbf{H}$ The Kaplan-Meier plot represents the survival time of BALB/C nude mice-bearing orthotopically implanted $5 \times 10^{5} \mathrm{Pt \# 3}$ cells stably expressing either LINC00461 or empty vector control (pcDNA3.1) ( $n=8-9$ per group). Log-rank test. The results are shown as mean \pm SEM for triplicate samples in each group
} 

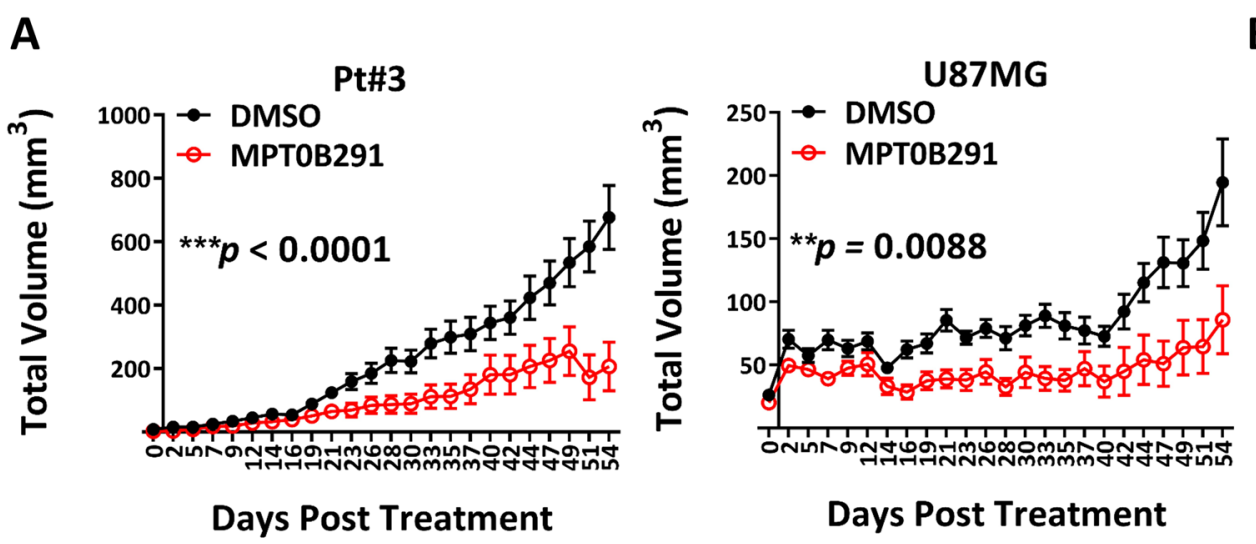

B

C

Pt\#3

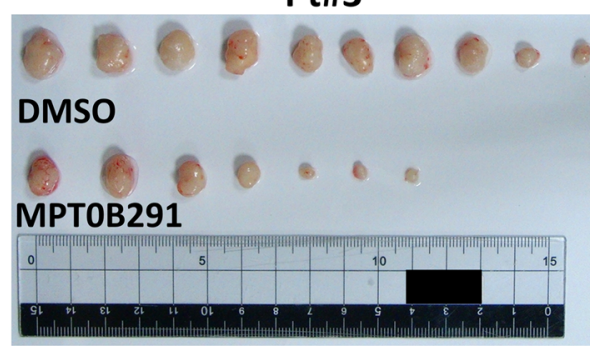

Days Post Treatment

U87MG
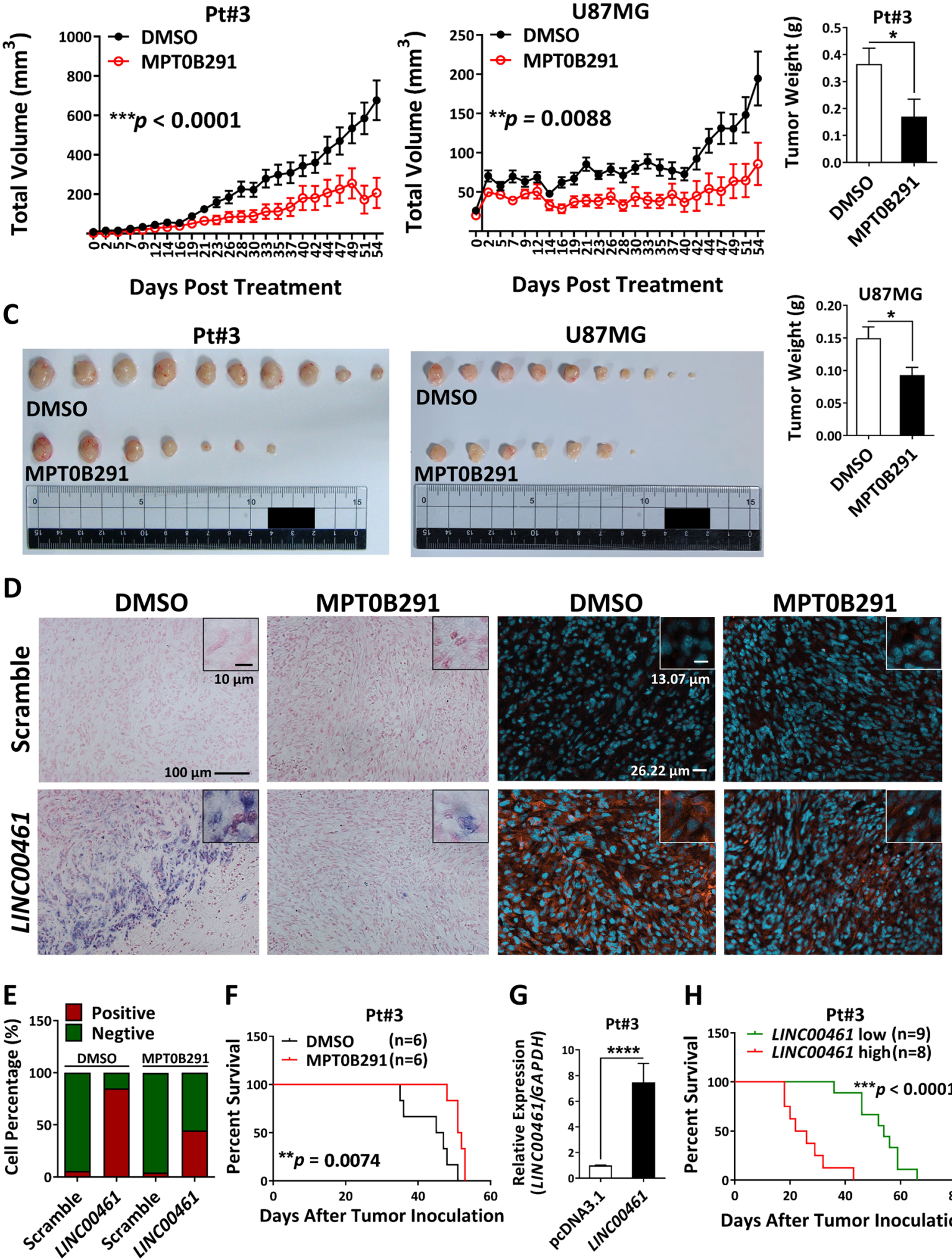

$\mathbf{F}$

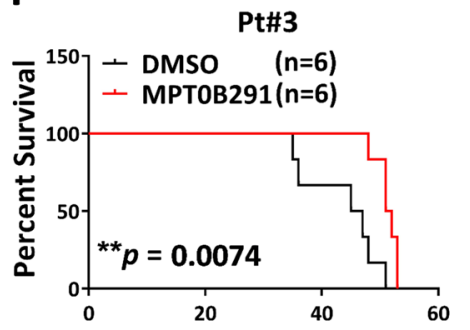

Days After Tumor Inoculation
G

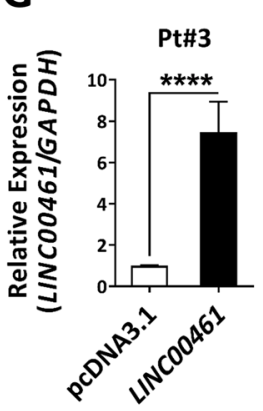

H

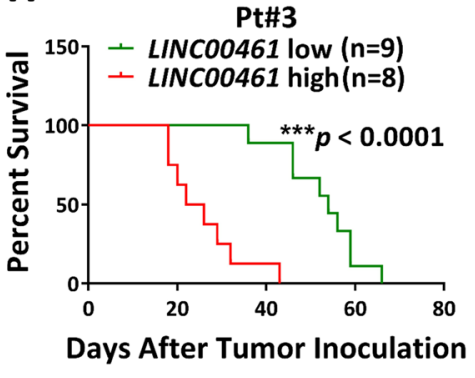

Fig. 2 (See legend on previous page.) 
and trichostatin $\mathrm{A}$, led to a decrease in the interaction of HDAC6 and CNOT6 in cells (Fig. S3A), suggesting that deacetylase activity is essential for HDAC6 to bind CNOT6, causing changes in the deadenylase activation. Apart from RNA-degrading enzymes that contribute to RNA degradation, RNA-protective proteins that contribute to the stability of LINC00461 were also investigated. We purified LINC00461 (Fig. 3G) and performed RNA-protein pull-down assay (Fig. S3B) combined with iTRAQ-based proteomic analysis, by which several LINC00461-binding proteins were identified (Fig. 3H). Further investigation of the proteomics data using IPA, two RNA-stabilizing proteins, FUS and vimentin, were discovered as HDAC6-targeted factors that functioned in regulating RNA stability. Interestingly, a previous study has indicated that FUS is associated with several RBPs in the vicinity of the mRNA $3^{\prime}$ end and it controls polyadenylate tail maintenance [23]. To investigate the role of HDAC6 on these RNA-regulating proteins, HDAC6 inhibition was induced by MPT0B291 treatment or HDAC6 knockdown and this caused a significant decrease in FUS expression in both parental and TMZ-resistant GBM cells and a significant increase in CNOT6 expression (Fig. 3I-J and Fig. S3C). Furthermore, the effect of FUS knockdown on the RNA stability of LINC00461 showed a 3-fold reduction in the half-life of LINC00461 in TMZresistant Pt\#3 cells (Fig. 3K).

\section{LINC00461 depletion suppresses GBM cell proliferation through cell-cycle arrest}

We examined the effects of LINC00461 on GBM cell proliferation. LINC00461 knockdown in parental and TMZ-resistant GBM cells reduced LINC00461 expression approximately by $40-50 \%$ (Fig. $4 \mathrm{~A}$ ). Interestingly, LINC00461 depletion suppressed cell proliferation significantly in both parental and TMZ-resistant GBM cells
(Fig. 4B). To further determine the anti-proliferative effect of LINC00461 silencing on GBM cells, we analyzed cell-cycle distribution by flow cytometry. LINC00461 knockdown induced a significant cell-cycle shift from G2/M to G1 phases in parental GBM cells and similarly induced a slight perturbation of cell-cycle progression into G2/M phase in TMZ-resistant cells (Fig. 4C). Recently, topoisomerase II alpha (TOP2A), a key enzyme in DNA replication to maintain cell proliferation, was found to be upregulated by LINC00461 through sponging of miR-411-5p during gliomagenesis [13]. Thus, we examined TOP2A expression, including levels of RNA (Fig. 4D) and protein (Fig. 4E), in our experimental model. The results showed that LINC00461 knockdown significantly attenuated TOP2A expression at the transcriptional level in both parental and TMZ-resistant U87MG cells. Additionally, MPT0B291 treatment showed a similar trend with reduced mRNA and protein expression of TOP2A in these GBM cells (Fig. 4F-G).

\section{LINC00461 is involved in HDAC6-mediated cell division and survival regulation}

To characterize the role of LINC00461 in GBM cells, we performed a genome-wide analysis of gene expression in LINC00461-silenced U87MG cells, and identified that 115 genes were upregulated and 219 genes were downregulated (Fig. 5A). Interestingly, the 219 down-regulated genes included TOP $2 A$ and $C C N A 2$, both containing a gene expressing cyclin A2 to maintain the G2/M transition phase of the cell cycle. A comprehensive analysis conducted by comparing the LINC00461-silenced dataset with the MPT0B291-treated dataset revealed 105 intersection genes (Fig. 5B). The expression of 88 genes showed the same trend in both treatments. Functional analysis by IPA revealed highly significant functional gene classes associated with "cell division (50 of

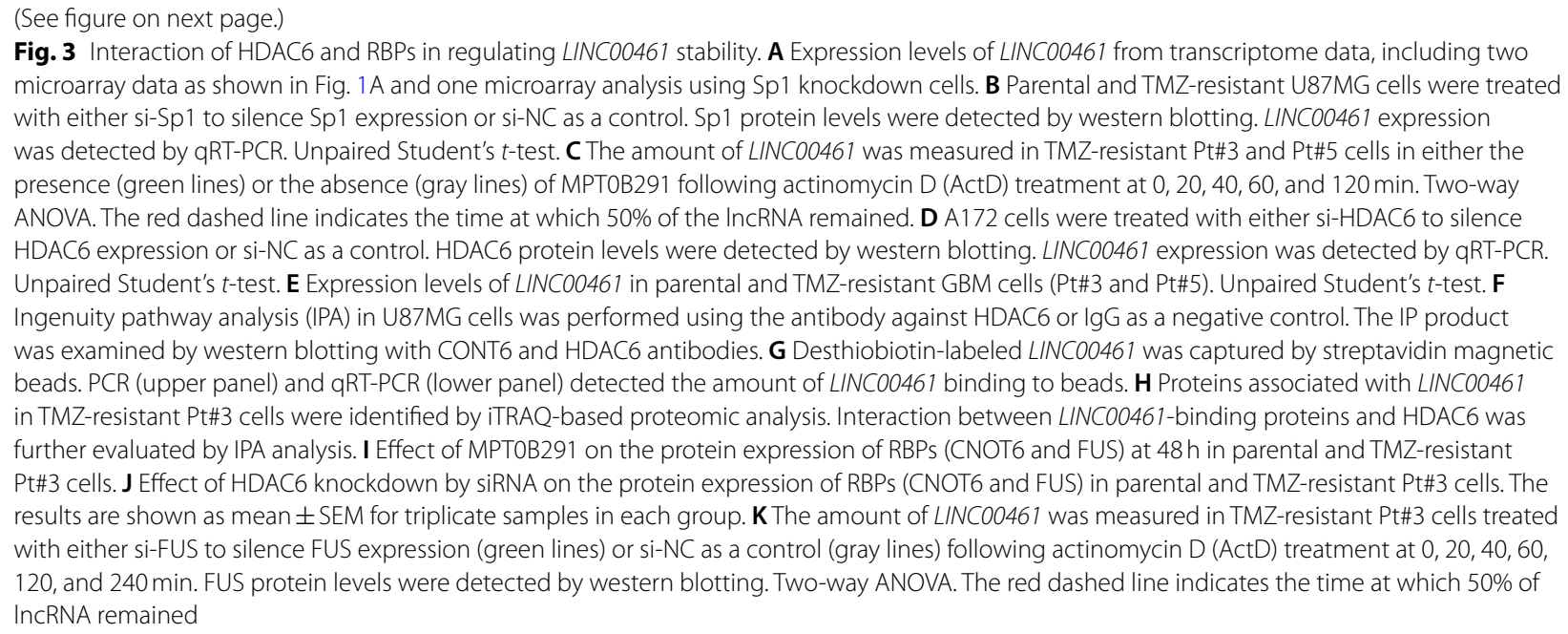




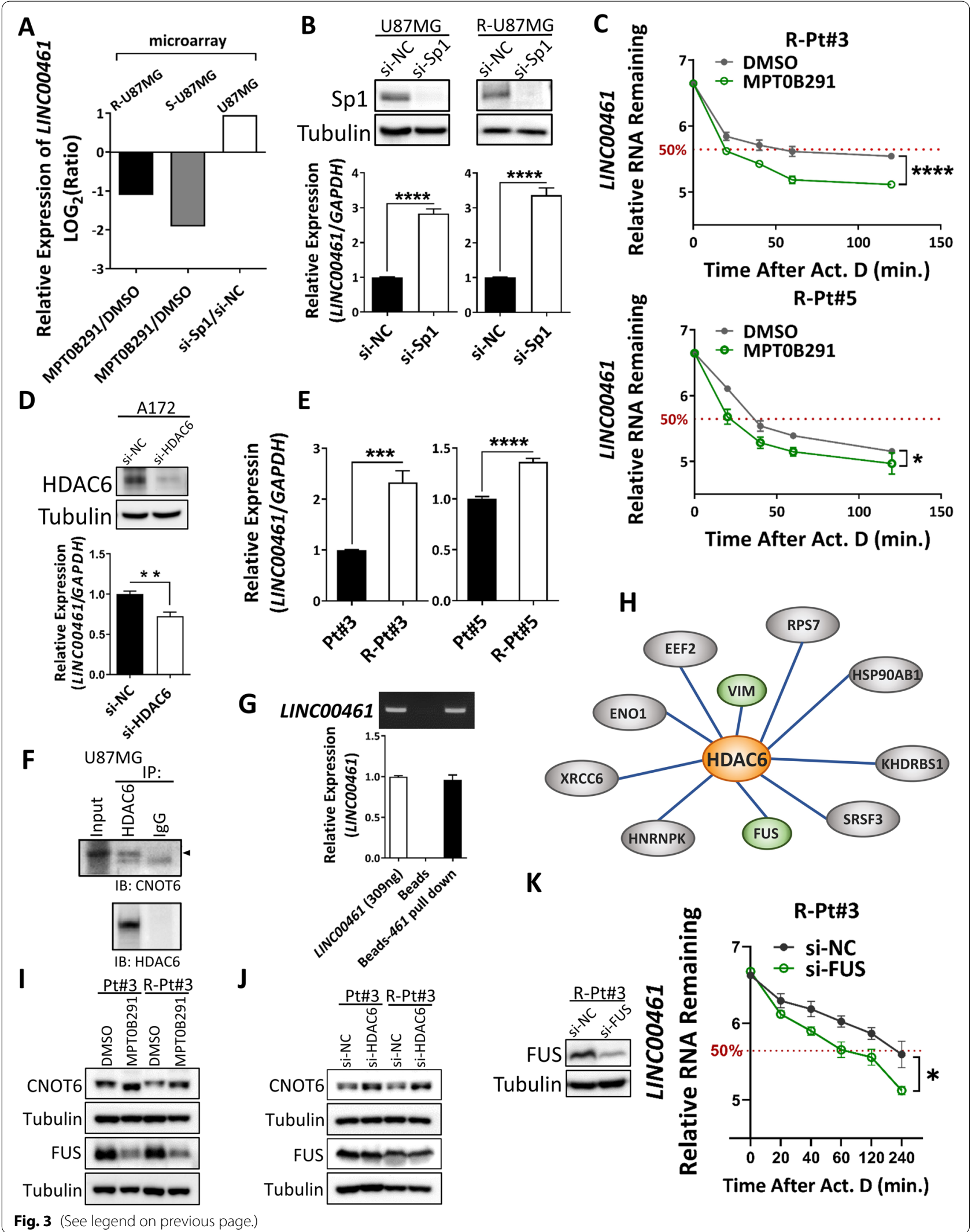


88 genes)" and "survival (49 of 88 genes)" (Fig. 5C). Furthermore, we examined the activity of several pathways using IPA, and found that either LINC00461 depletion or MPT0B291 treatment significantly inhibited cell proliferation/survival and induced cell death/senescence in GBM (Fig. 5D). These data indicated that LINC00461 is involved in HDAC6-regulated GBM malignancy. To further confirm the clinical relevance of the 50 cell-divisionrelated genes appearing in the intersection dataset $(88$ genes), we analyzed the TCGA-GBM next-generation sequencing (NGS) datasets of 124 patients. A strong negative correlation was observed (PCC: - 0.452) (Fig. 5E). The result indicated that abnormalities in the expression of the 50 cell-division-related genes are inverted following the HDAC6/LINC00461 inhibition in GBM. Moreover, survival analysis using clinical outcome data showed that several cell-division-related genes (CENPF, DLGAP5, GTSE1, HMMR, KIF14, MCM10, MELK, RACGAP1, TMPO, and TOP2A) have poor prognostic implications in high-grade gliomas (Fig. 5F and Fig. S4A), suggesting that the reducing expressions of these genes through HDAC6/LINC00461 inhibition may improve outcomes in GBM patients. Additionally, the importance of the HDAC6/LINC00461-mediated regulation in clinical GBM was analyzed using single-cell transcriptome sequencing datasets. Surprisingly, higher expressions of LINC00461 and its associated downstream cell-divisionrelated genes mentioned in Fig. $5 \mathrm{E}$ were enriched in a specific cluster (Cluster 5) of GBM cells (Fig. 5G-H). Consistently, functional analysis of the conserved markers in Cluster 5 by IPA indicated highly similar functional gene classes as that of the LINC00461-silenced and MPT0B291-treated datasets mentioned in Fig. 5C (Fig. S5A, Table S4).

\section{LINC00461 depletion downregulates cell-cycle-related protein MELK by blocking the interaction between LINC00461 and miR-485-3p}

We further investigated downstream targets of LINC00461, especially in cell-cycle-related proteins. Either MPT0B291 treatment or LINC00461 depletion significantly downregulated the expression of several cell-division-regulated factors, including Ki67, maternal embryonic leucine zipper kinase (MELK), DLG associated protein 5 (DLGAP5), hyaluronan mediated motility receptor (HMMR), minichromosomal maintenance protein 10 (MCM10), and thymopoietin (TMPO) (Fig. 6AB). Given that LINC00461 is dominantly located in the cytoplasm [13], we explored its function as a miRNA sponge to repress the inhibitory effect of miRNAs on target mRNA. Prediction of the lncRNA-miRNA-mRNA networks (Fig. S6A) using three public databases (miRDB [24], miRWALK [25], and starBase [26]) revealed 13 intersecting miRNAs in the datasets (Fig. S6B). Of these, miR-485-3p, a tumor suppressor in GBM [27] was presumed to inhibit MCM10 and MELK. Thus, we examined whether LINC00461 indeed played a role in sponging miR-485-3p. MPT0B291 treatment-mediated LINC00461 inhibition led to significantly elevated miR485-3p expression (Fig. 6C) but it significantly reduced MELK expression in parental and TMZ-resistant GBM cells (Fig. 6D). However, the simultaneous overexpression of LINC00461 in cells decreased the induction of miR485-3p levels (Fig. 6C) and reduction of MELK expression by MPT0B291 (Fig. 6D), suggesting that LINC00461 maintains MELK expression by sponging miR-485-3p.

\section{Discussion}

Although lncRNAs were considered non-functional and transcriptional noise initially, cumulative evidence over the past decade has unraveled that IncRNAs perform diverse biological functions [28]. Strategies for targeting lncRNAs in cancer treatment have gained widespread interest because they have vital roles in gene regulation and thus modulate hallmark processes of cancer [29]. Our previous studies demonstrated that HDAC inhibition using inhibitors represses stem-like properties in GBM that provide a potential therapeutic strategy to overcome drug resistance [10, 30]. Nevertheless, those two studies focused on HDAC-regulated protein-coding genes associated with drug resistance, but the role of HDACs on the impact of dysregulated lncRNAs remains

\footnotetext{
(See figure on next page.)

Fig. 4 Inhibition of LINCO0461 downregulates TOP2A, resulting in the blocking of cell proliferation and cell-cycle progression in GBM cells. A Expression levels of LINC00461 in parental and TMZ-resistant GBM cells (U87MG and A172) treated with either si-NC or si-LINC00461. Unpaired Student's $t$-test. B Effect of LINC00461 depletion on proliferation in parental and TMZ-resistant GBM cells (U87MG and A172). The x-axis indicates the time after siRNA transfection. $\mathrm{OD}_{550}$ values relative to si-NC on day 0 are shown in the $y$-axis. Two-way ANOVA. C Cells (U87MG and R-U87MG) with or without LINC00461 knockdown were fixed and stained with propidium iodide, and then their DNA contents were measured by flow cytometry. $X$ - and $y$-axes denote DNA content and cell number, respectively. The lower panel shows the quantitative measurements of each of cell-cycle phase. Unpaired Student's t-test. D Expression of TOP2A mRNA in U87MG and R-U87MG cells that were treated with either si-NC or si-LINC00461. Unpaired Student's t-test. E Cells (U87MG and R-U87MG) were treated with si-NC or si-LINC00461. After knockdown, TOP2A protein levels were detected by western blotting. Quantification of TOP2A expression from triplicate samples is shown below. Unpaired Student's t-test. F and G Cells (U87MG and R-U87MG) were treated with different doses of MPTOB291 for $2 \mathrm{~d}$, and the levels of TOP2A mRNA (F) and protein $(\mathbf{G})$ in cells were then detected by qRT-PCR and western blotting, respectively. Quantification of mRNA and protein levels was obtained from triplicate samples. One-way ANOVA. The results are shown as mean \pm SEM for triplicate samples in each group
} 


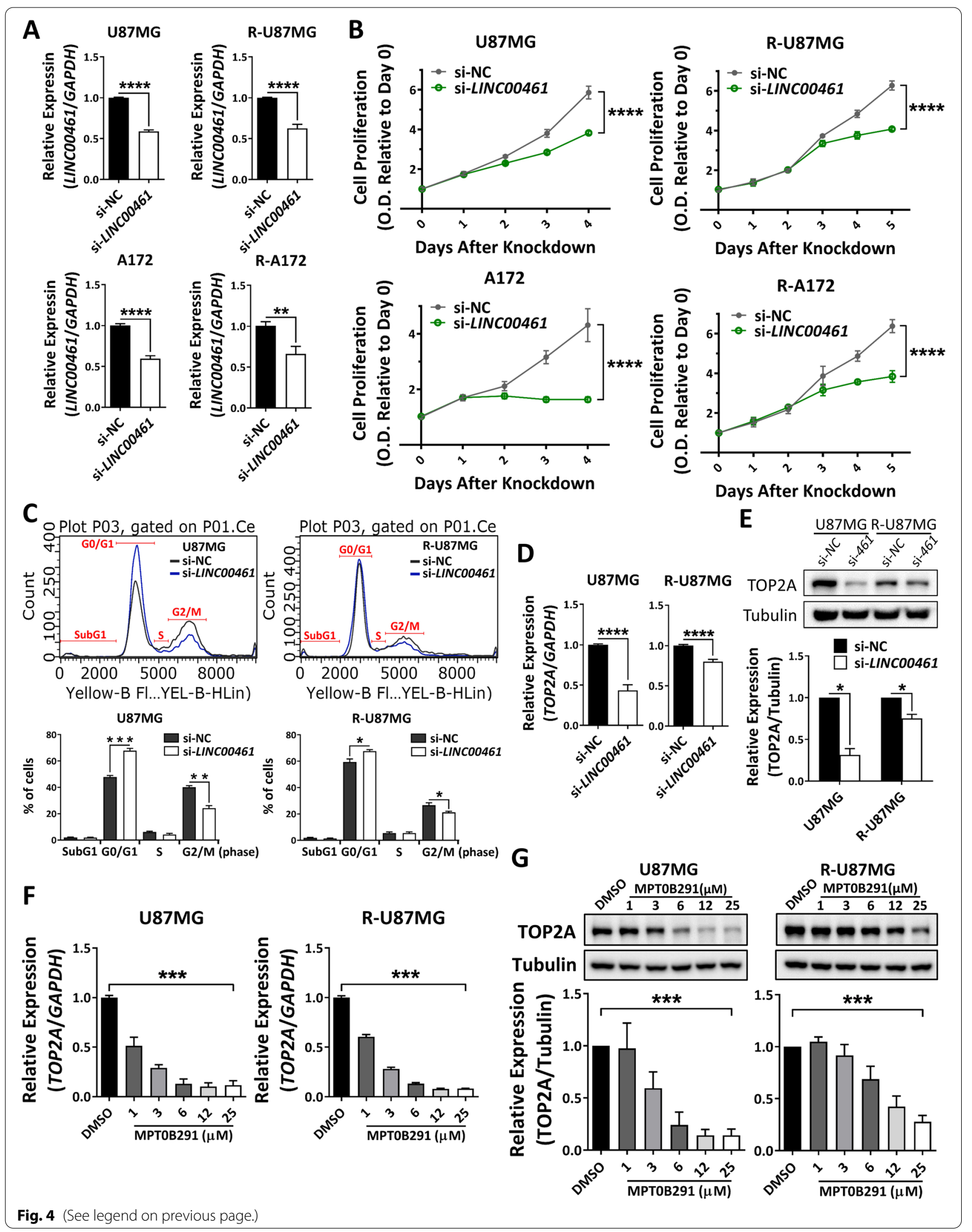


unclear and hence needs further investigation. Here, we investigated the involvement of HDAC6 in LINC00461 regulation by performing microarrays and RNA-seq analysis in stem-like and TMZ-resistant GBM cells with HDAC6 inhibition using MPT0B291 and siRNA.

According to a transition in investigative approaches from lncRNA annotation and molecular characterization to tumorigenic mouse models needed for deciphering the physiological roles of cancer-associated lncRNAs [29], we examined the impact of LINC00461 on GBM growth and survival in vivo. Evidence gathered shows that MPT0B291 treatment significantly reduced subcutaneous tumor growth along with a decreased level of LINC00461 and a negative correlation between LINC00461 expression and survival of mice with intracerebral GBM, suggests a pivotal role for LINC00461 as an oncogenic factor in GBM malignancy. Indeed, a previous study has demonstrated that the expression of C130071C03Rik, the mouse ortholog of LINC00461, is increased not only in precancerous conditions but also during glioma progression [13]. Some characteristics suggest that LINC00461 is a potentially promising target for treating patients with GBM. First, previous literature on LINC00461 demonstrated its oncogenic functions across diverse cancer types [13, 14, 31, 32]. Second, LINC00461 displays a brain-specific expression pattern, which implies reduced unintended toxic effects associated with their targeting. Third, unlike most intergenic IncRNAs that show poor sequence conservation, LINC00461 has a high degree of sequence homology across vertebrates [13], suggesting its essential role among such species [33].

The mechanisms underlying the regulation of mRNA stability have been extensively reviewed [34], whereas little is known about the modulation of lncRNA stability. Considering the inhibitory potency of MPT0B291 on HDAC 1 and 2 ( $\mathrm{IC}_{50} \approx 0.31$ and $1.16 \mu \mathrm{M}$, respectively) [10, 11], the impact of HDAC1 and HDAC2 on LINC00461 expression was also examined in GBM cells using
siRNA-mediated depletion. Interestingly, the silencing of neither HDAC1 nor HDAC2 was involved in modulating LINC00461 expression (Fig. S7A), suggesting that a decrease in LINC00461 expression by MPT0B291 is mainly due to the inhibition of HDAC6 rather than that of HDAC1 or HDAC2. Although several aspects discriminate lncRNAs from protein-coding mRNA, they also share certain similarities [35]. Numerous lncRNAs are subjected to transcriptional editing, for example, splicing, polyadenylation, and $5^{\prime}$ capping, just like mRNAs [35]. Post-transcriptional regulation of lncRNAs is mediated by RBPs in mainly two aspects: stability and localization. Our results identified two HDAC6-associated proteins (CNOT6 and FUS) that might explain a shorter half-life of LINC00461 in response to MPT0B291 treatment (Fig. S7B). Because the enzyme activity of CNOT6 is required for deadenylation by the CCR4-NOT nuclease complex [36], and acetylation enhances CNOT6 activity inducing RNA degradation [22], we predict that high levels of HDAC6 in GBM cells inhibit CNOT6 acetylation, leading to LINC00461 stabilization, while MPT0B291 treatment induces CNOT6 acetylation, promoting LINC00461 degradation (Fig. S7B). FUS-containing 3 '-end processing machinery facilitates mRNA stability [23], and lysine status regulated by cyclic adenosine monophosphate-response element binding protein/p300 and histone deacetylase families contribute to FUS function [37]. Therefore, in GBM cells, high levels of HDAC6 may increase the RNA-binding affinity of FUS, resulting in LINC00461 stabilization, whereas MPT0B291 treatment induces FUS acetylation, promoting LINC00461 degradation (Fig. S7B). Although our current results prove that HDAC6 inhibition increases CNOT6 and decreases FUS protein levels, further studies are needed to confirm whether HDAC6 directly regulates the lysine status of these two proteins.

Although recent studies have shown the oncogenic roles of LINC00461 in tumors [13, 14, 31, 32],

\footnotetext{
(See figure on next page.)

Fig. 5 Involvement of LINC00461 in HDAC6-mediated cell division and survival in GBM cells. A Volcano plot visualizing the differentially expressed genes between si-NC and si-LINC00461 (si461) treatment. Green and red dots represent genes with significant downregulation and upregulation ( $p$-value $<0.01$ ), respectively, in si-LINC00461 compared with si-NC. B Venn diagram illustrating overlaps between the number of genes that were significantly altered in MPTOB291-treated and si-LINC00461-treated cells. C Top five molecular and cellular functions of genes altered in si-LINC00461-treated (334 genes), MPTOB291-treated (1709 genes), and intersection subset (88 genes) by IPA. The number of genes involved in the biological process is shown on the right side of the bar chart. $\mathbf{D}$ Heatmap representation of the top 20 predicted influential biological functions after treatment by IPA. Positive activation Z-score (red) represents activated pathways, and negative activation Z-score (green) represents inhibited pathways. E Relationships between si-LINC00461-treated NGS data (in horizontal) and TCGA-GBM NGS data (in vertical) by Pearson's correlation coefficient (PCC). Each dot represents the expression value of a cell-division-related gene that existed in the intersection subset. F Forest plots showing hazard ratios for risk of mortality in high-grade glioma patients with higher expressions of the indicated genes. Circles represent the hazard ratio, and bars on both sides denote their corresponding $95 \%$ confidence intervals (Cl). The original survival curves were obtained from PROGgeneV2 (Fig. S4). Log-rank test. G Individual cells suspended/isolated directly from patient's fresh GBM sample were used for single-cell RNA sequencing. Each dot denotes one cell with the expression of LINC00461, and the depth of blue represents the amount of LINC00461 expression. The red dashed circle indicates cluster 5. H Expression profiles of LINCO0461 and cell-division-related genes in cell clusters from the single-cell RNA sequencing dataset. Dot size reflects the percent of cells expressing the gene, and dot color indicates average gene expression
} 


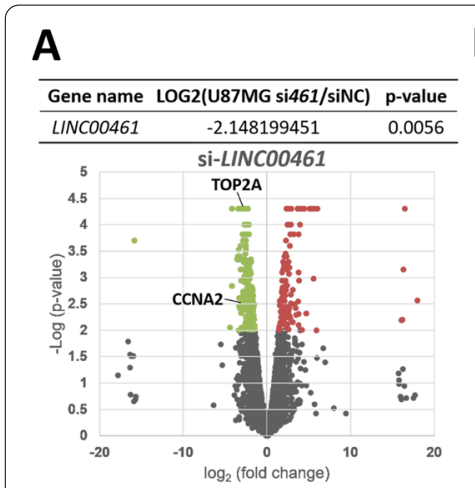

B

D

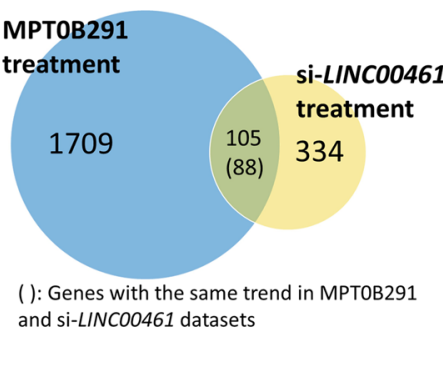

E
C

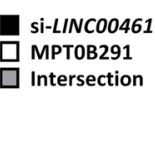

Cell Death and Survival-

Top enriched biological functions

DNA Replication Recombination and Repai

Cellular Movement

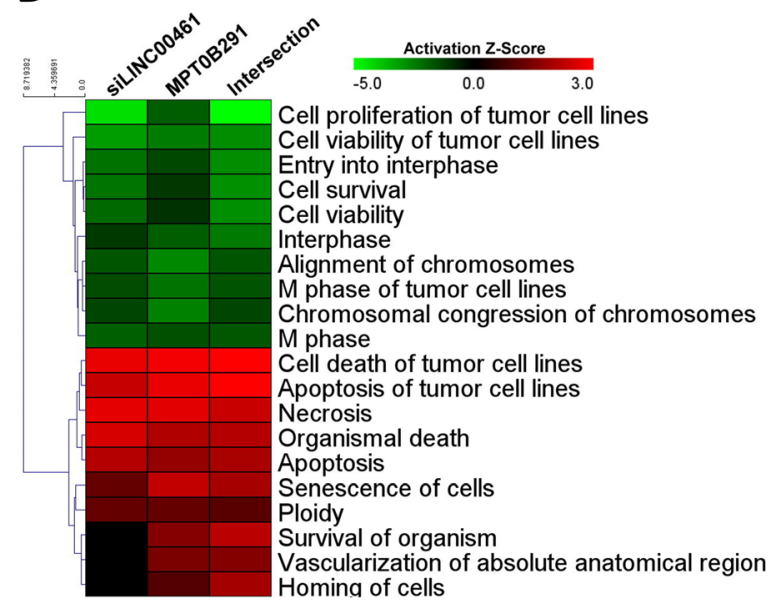

Homing of cells

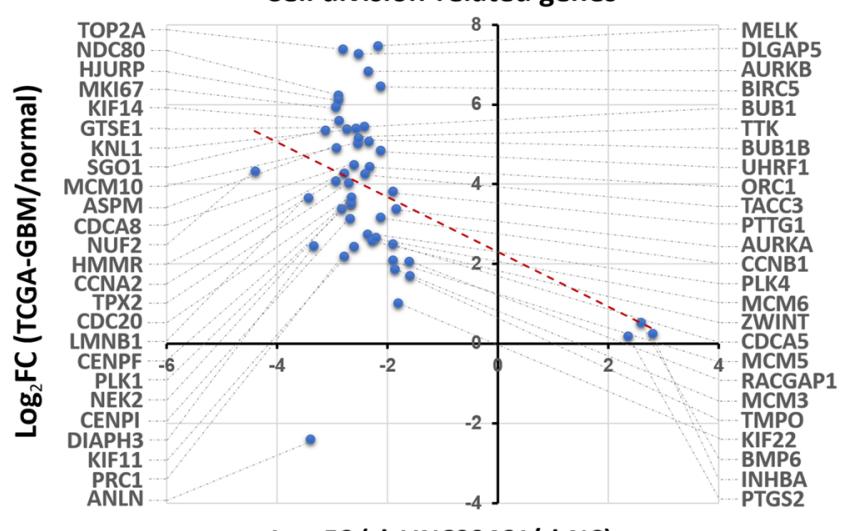

$\log _{2} \mathrm{FC}$ (si-LINCO0461/si-NC)
$\mathbf{F}$

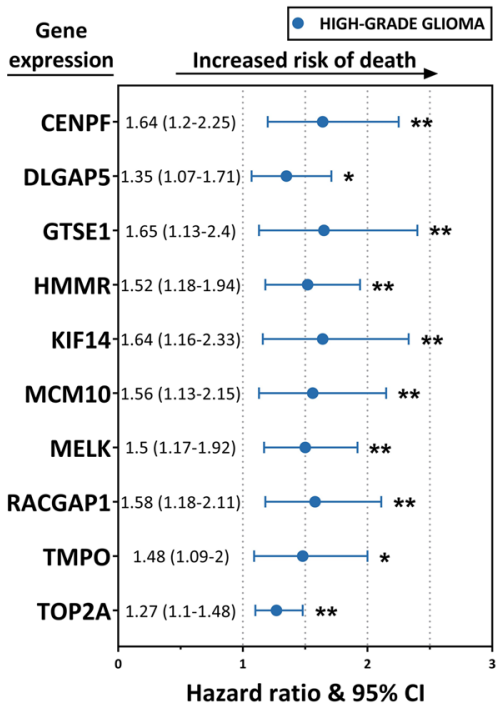

G

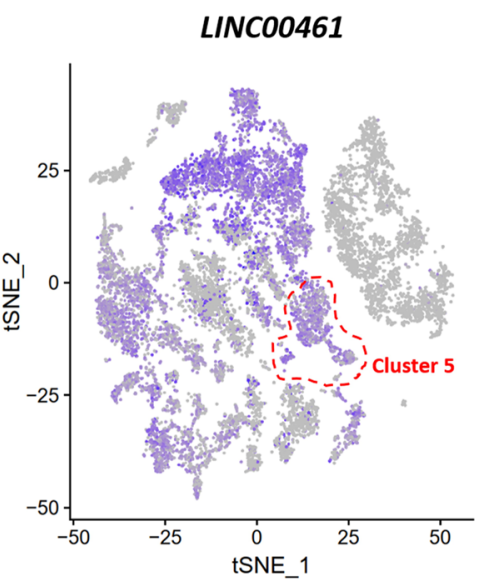

H Percent Expressed Average Expression

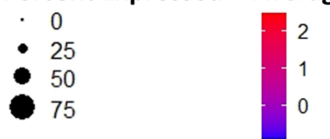

Features

Fig. 5 (See legend on previous page.) 
the molecular mechanisms underlying LINC00461 regulation remain unclear. To understand the function of LINC00461 comprehensively, we compared the LINC00461-silenced dataset with the clinical TCGAGBM dataset. Pathway enrichment analysis of intersected genes in these two datasets revealed cell growth, which is consistent with the findings that LINC00461 promoted GBM proliferation through the regulation of TOP2A expression. TOP2A is a nuclear enzyme that controls and alters the topologic states of DNA during transcription and mitosis, facilitating gene expression and mitotic progression, respectively, in tumor cells [38]. Here, we found that the HDAC6/LINC00461 axis inhibition downregulated TOP2A expression in parental and TMZ-resistant cells, thereby explaining the perturbation of cell-cycle progression into G2/M phase. In addition, a previous study has demonstrated that LINC00461 knockdown reduces expressions of a few cell-division-related genes, including cyclin D1, CDK4, cyclin A2, and cyclin $\mathrm{E}$ in U87MG [39]. Although genome-wide analysis with critical criteria for selecting potential LINC00461 downstream targets from altered genes in the LINC00461silencing dataset only identified cyclin A [39] and TOP2A [13] as presented previously, we measured the expressions of the other cell-division-related genes mentioned above in our GBM cell model. Significant decreases in the expression levels of these cell-division-related genes (cyclin D1, CDK4, cyclin A2, and cyclin E1) following LINC00461 knockdown were observed in parental and TMZ-resistant U87MG (Fig. S8A). Furthermore, LINC00461 knockdown significantly downregulated protein expressions of cyclin D1 and CDK1 (Fig. S8B), which is consistent with the findings of a previous report [39]. Except for the LINC00461-regulated cell-cycle-related genes, which have already been proved, numerous celldivision-related genes that are modulated by LINC00461 were revealed in this study for the first time (Figs. 5E and 6). In summary, LINC00461 displays a widespread regulation of cell-division-related proteins that are indispensable for GBM growth.

Apart from exploring the function of LINC00461 using the public clinical dataset, we also performed transcriptome profiling of clinical samples at the single-cell level.
Recent advances in the development of NGS technologies have overcome conventional profiling methods that assess bulk populations and provide the opportunity to gain insights into the characterization of individual cells [40]. Considering the heterogeneity of tumor biology, we explored whether there is a cluster of cells overexpressing LINC00461. Surprisingly, our findings revealed that the cells in Cluster 5 showed highly elevated expressions of LINC00461 and cell-division-related genes simultaneously and also displayed biomarkers enriched in pathways associated with cell division (Fig. S5A, Table S1). Therefore, the cell characteristics in Cluster 5 are require further study to clarify the clinical malignancy of GBM and the development of treatment strategies.

Like proteins, appropriate subcellular localization patterns of lncRNAs allow for the primary determination of their molecular functions [41]. Our observations in xenograft tumors through both RNA-ISH and RNAFISH staining demonstrated that LINC00461 localizes to the cytoplasm rather than to the nucleus. Cytoplasmic lncRNAs modulate mRNA fate by acting as miRNA sponges that compete for miRNA binding [42]. To dissect the regulatory mechanism between LINC00461 and cell-division-related proteins comprehensively, we used three reliable public datasets to predict lncRNAmiRNA-mRNA networks. Downregulation of MCM10 and MELK proteins by LINC00461 depletion combined with the induction of miR-485-3p by LINC00461 inhibition using MPT0B291 suggests that this strategy is effective for identifying the downstream regulatory networks of LINC00461.

\section{Conclusions}

Recent research has unraveled the indispensable roles of lncRNAs in regulating GBM malignancy, including cancer stem-like features [8]. Nevertheless, the mechanism by which cancer-associated lncRNAs are dysregulated remains unexplored. Here, our results provide an insight into lncRNA regulation by HDAC proteins and lead to a comprehensive understanding of LINC00461associated networks in GBM malignancy, by expounding the utility of the HDAC6/RBPs/LINC00461 axis as a potential therapeutic approach for treating patients with

\footnotetext{
(See figure on next page.)

Fig. 6 LINC00461 maintains the expression of cell-cycle-related proteins by sponging tumor-suppressive miRNAs. A Effect of MPTOB291 on the protein expression of cell-division-related genes at $48 \mathrm{~h}$ in parental and TMZ-resistant Pt\#3 cells. Quantification of expression from triplicate samples is shown alongside the data. Unpaired Student's t-test. B Effect of LINC00461 depletion on the protein expression levels of cell-division-related genes in parental and TMZ-resistant U87MG cells. Quantification of expression from triplicate samples is shown alongside the data. Unpaired Student's $t$-test. C The combined effect of LINC00461 elevation and MPTOB291 treatment on the expression of miR-485-3p in parental and TMZ-resistant U87MG cells. Unpaired Student's $t$-test. D The combined effect of LINC00461 elevation and MPTOB291 treatment on the expression of MELK in parental and TMZ-resistant U87MG cells. Unpaired Student's t-test. E A schematic diagram illustrating how the HDAC6/RBP/LINC00461 axis maintains the expression of cell-cycle-related protein MELK by sponging tumor-suppressive miR-485-3p, but the HDAC6 inhibition induced by MPT0B291 is able to block LINC00461 functions, reducing GBM malignancy. The results are shown as mean \pm SEM for triplicate samples in each group
} 


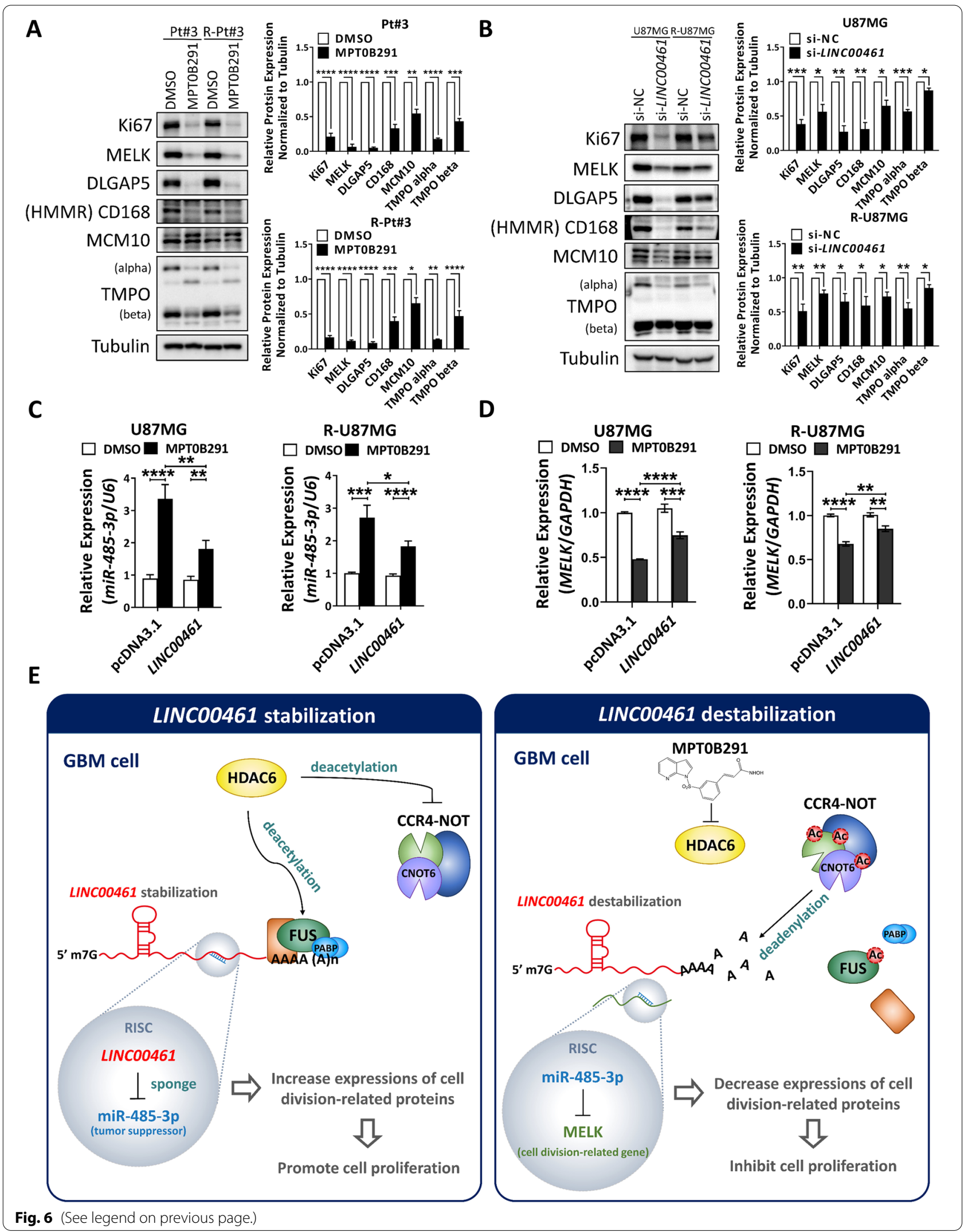


GBM (Fig. 6E). However, this research has thrown up many questions that need further investigation. Future research should focus on the identification of the lysine residue of RBPs that is deacetylated by HDAC6, isolating the LINC00461-miRNA-mRNA axis that is the most important compared to the others, and understanding the mechanism of targeting LINC00461-overexpressed cells effectively in clinical practice.

\begin{abstract}
Abbreviations
ActD: Actinomycin D; AP: Alkaline phosphatase; AR: Androgen receptor; CNOT6: CCR4-NOT core exoribonuclease subunit 6; Cl: Confidence interval; DIG: Digoxigenin; DLGAP5: DLG associated protein 5; ECL: Enhanced chemiluminescence; FISH: Fluorescence in situ hybridization; FUS: Fused in sarcoma; GBM: Glioblastoma; GEO: Gene expression omnibus; HDAC: Histone deacetylase; HMMR: Hyaluronan mediated motility receptor; IPA: Ingenuity pathway analysis; ISH: In situ hybridization; ITGB3: Integrin $\beta 3$; IncRNAs: Long non-coding RNAs; TRAQ: Isobaric tags for relative and absolute quantitation; MCM10: Minichromosomal maintenance protein 10; MELK: Maternal embryonic leucine zipper kinase; MPTOB291: Azaindolylsulfonamide compound 12; MTT: 3-(4,5-dimethylthiazol-2-yl)-2,5-diphenyltetrazolium bromide; NGS: Next-generation sequencing; PCC: Pearson's correlation coefficient; RBPs: RNA-binding proteins; scRNA-seq: Single-cell RNA sequencing; SNR: Signalto-noise-ratio; TCGA: The cancer genome atlas; TMPO: Thymopoietin; TMZ: Temozolomide; TOP2A: Topoisomerase II alpha; TRITC: Tetramethylrhodamineisothiocyanate; TSA: Trichostatin A.
\end{abstract}

\section{Supplementary Information}

The online version contains supplementary material available at https://doi. org/10.1186/s13046-022-02257-w.

Additional file 1: Figure S1. MPTOB291 commonly alters two IncRNAs (LINC00461 and LINC01559) in stem-like and TMZ-resistant U87MG cells. Volcano plots show overlapping IncRNAs between two microarray data (MS1040791 and MS1060987). Relative expression level (log2) to control (DMSO) of each IncRNA is shown in the $x$-axis. $y$-axis indicates SNR (signalto-noise ratio) value (log2) of each IncRNA. Genes with significant upregulation (red) and downregulation (green) in both microarray datasets are highlighted. Figure S2. MPTOB291 decreases Ki-67 expression in vivo. (A) IHC detected the protein expressions of proliferation (Ki-67) and apoptosis (cleaved caspase-3) marker. Serial sections of control and MPTOB291treated xenografts were hybridized with either an antibody against Ki-67 (upper two panels) or cleaved caspases-3 (lower two panels), and cell nuclei were stained with hematoxylin. Scale bars indicate $20 \mu \mathrm{m}$ and $200 \mu \mathrm{m}$. Mean intensity of Ki-67 and cleaved caspases-3 staining in the cells of control and MPTOB291-treated xenografts were semi-quantitative determined. Unpaired Student's $t$-test. Figure S3. Interaction between HDAC6 and RNA-binding proteins (CNOT6 and FUS). (A) Flag-tagged CNOT6-expressed U87MG cells were treated with 10 $\mu$ M MPT0B291, $10 \mu \mathrm{M}$ Trichostatin A (TSA), or DMSO for $2 \mathrm{~h}$. Flag-CNOT6 protein was immunoprecipitated and the precipitated samples were then analyzed by immunoblotting analysis with antibodies of HDAC6 or Flag-M2. (B) Enrichment of HuR from the lysate by desthiobiotin-labeled androgen receptor (AR) 3'-UTR validates the efficiency of RNA-protein pull-down assay (left panel). Protein lystaes from TMZ-resistant Pt\#3 cells were also used for the RNAprotein pull-down assay with desthiobiotin-labeled LINC00461, and both poly(A)-binding protein (PABP) and HuR were slightly detected in the pulldown sample, but desthiobiotin-labeled LINC00461 did not bind HDAC6 directly. $(\mathrm{L}=$ lysate; $\mathrm{FT1}=$ flow-through in first wash; $\mathrm{FT} 2=$ flow-through in second wash; $E=$ eluate). (C) Quantification of CNOT6 and FUS protein expression from the parental and TMZ-resistant Pt\#3 cells either treated with MPTOB291 or si-HDAC6. Unpaired Student's t-test. The results are shown as mean \pm SEM for triplicate samples in each group. Figure S4. Cell division-related genes show poor prognostic implications in high-grade glioma patients. (A) Kaplan-Meier curves compare the survival outcomes in high-grade glioma patients with high (red) and low (green) expressions of cell division-related genes. All survival curves were obtained from PROGgeneV2. Log-rank test. Figure S5. Functional analysis of the conserved markers in cluster 5 from scRNA-seq data reveals gene classes associated with cell division and survival. Core analysis using IPA revealed the top ten molecular and cellular functions of highly conserved marker genes in cluster 5 of patient-derived GBM cells. Log( $p$-value) indicates the significance of enrichment for highly expressed marker genes from our dataset. The threshold for significance was set at a $p$-value $<0.05$. Figure S6. Three datasets identify potential interaction networks between 13 miRNAs and cell division-related genes. (A) Pipeline for the identification of potential regulatory LINC00461-miRNA-mRNA networks. (B) The miRNAmRNA networks were identified using three public databases (miRDB, miRWALK, and starBase). Figure S7. The regulation of LINC00461 stability. Protein expression levels of HDAC1 and HDAC2 in parental and TMZ-resistant U87MG cells treated with either si-NC or si-HDAC1/2. Effect of either HDAC1 or HDAC2 depletion on LINC00461 expression in parental and TMZ-resistant U87MG cells. n.S., not significant, unpaired Student's t-test. (B) A schematic diagram illustrates the proposed regulatory mechanism underlying HDAC6 controls the LINC00461 stability via regulating both the RNA-binding activity of FUS (fused in sarcoma)/PABP and the activity of deadenylases of human Ccr4-Not complex. Figure S8.LINC00461 knockdown downregulates the expressions of cell division-related molecules. (A) Expressions of the cell division-related mRNAs (cyclin D1, CDK4, cyclin A2, cyclin E1, MELK, and MCM10) in parental and TMZ-resistant R-U87MG cells that were treated with either si-NC or si-LINC00461. Unpaired Student's t-test. (B) Effect of LINCO0461 depletion on the protein expression levels of cell division-related molecules (cyclin D1 and CDK1) in parental and TMZ-resistant U87MG cells. Table S1. siRNA targeted-sequences for each gene. Table S2. The primer or probe sequences for each gene used in SYBR green and TaqMan qPCR assays. Table S3. Custom LNA ${ }^{\text {TM }}$ detection probes sequences. Table S4. List of biomarkers with fold-change more significant than 1.5 in cluster 5 .

\section{Acknowledgments}

Not applicable.

\section{Authors' contributions}

Investigation and validation: A.C.W, W.B.Y., J.P.L., R.Y.S., and T.I.H. Clinical specimen collection: K.Y.C., J.S.L., and P.Y.C. Data curation: A.C.W., K.Y.C., S.M.C, D.Y.H., C.Y.W., P.Y.C., and J.Y.C. Supervision: U.K., W.C.C., P.Y.C., and J.Y.C. Funding acquisition, P.Y.C. and J.Y.C. Writing draft: A.C.W. and J.Y.C. All authors have revised and approved the final version of the manuscript.

\section{Funding}

This study was supported by the Ministry of Science and Technology in Taiwan (grant numbers 110-2636-B-038-004 and 109-2320-B-182A-005-MY3).

\section{Availability of data and materials}

All data supporting the findings of this study are available within the article and supplementary data. The RNA-seq data discussed in this publication have been deposited in NCBI's Gene Expression Omnibus (GEO) and are accessible through GEO Series accession number GSE182220 (https://www.ncbi.nlm.nih. gov/geo/query/acc.cgi?acc=GSE182220).

\section{Declarations}

Ethics approval and consent to participate

Experiments were conducted under a protocol approved by the Joint Institutional Review Board of the Taipei Medical University, Taiwan with the registration numbers (Nos. 201006011 and 201402018) and by the Institutional Animal Care and Use Committee of the National Health Research Institute, Taiwan with the registration number (NHRI-IACUC-106010).

\section{Consent for publication}

Not applicable. 


\section{Competing interests}

The authors declare that there are no potential conflicts of interest.

\section{Author details}

${ }^{1}$ Graduate Institute of Medical Sciences, College of Medicine, Taipei Medical University, Taipei, Taiwan. ${ }^{2}$ The Ph.D. Program for Neural Regenerative Medicine, Taipei Medical University, 250 Wuxing Street, Taipei 11031, Taiwan. ${ }^{3}$ TMU Research Center of Neuroscience, Taipei Medical University, Taipei, Taiwan. ${ }^{4}$ National Institute of Cancer Research, National Health Research Institutes, Tainan, Taiwan. ${ }^{5}$ Department of Neurosurgery, National Cheng Kung University Hospital, Tainan, Taiwan. ${ }^{6}$ School of Pharmacy, Taipei Medical University, Taipei, Taiwan. ${ }^{7}$ TMU Research Center of Cancer Translational Medicine, Taipei Medical University, Taipei, Taiwan. ${ }^{8}$ Cell Physiology and Molecular Image Research Center, Wan Fang Hospital, Taipei Medical University, Taipei, Taiwan. ${ }^{9}$ The Ph.D. Program for Cancer Molecular Biology and Drug Discovery, Taipei Medical University, Taipei, Taiwan. ${ }^{10}$ Department of Neurosurgery, Keelung Chang Gung Memorial Hospital, Chang Gung University, 222 Mai-jin Road, Keelung 20401 Taiwan. ${ }^{11}$ Department of Biomedical Science and Environmental Biology, Kaohsiung Medical University, Kaohsiung, Taiwan.

Received: 30 September 2021 Accepted: 17 January 2022

Published online: 02 February 2022

\section{References}

1. Stupp R, Hegi ME, Mason WP, van den Bent MJ, Taphoorn MJ, Janzer $\mathrm{RC}$, et al. Effects of radiotherapy with concomitant and adjuvant temozolomide versus radiotherapy alone on survival in glioblastoma in a randomised phase III study: 5-year analysis of the EORTC-NCIC trial. Lancet Oncol. 2009;10(5):459-66.

2. Brennan CW, Verhaak RG, McKenna A, Campos B, Noushmehr H, Salama SR, et al. The somatic genomic landscape of glioblastoma. Cell. 2013;155(2):462-77.

3. Verhaak RG, Hoadley KA, Purdom E, Wang V, Qi Y, Wilkerson MD, et al. Integrated genomic analysis identifies clinically relevant subtypes of glioblastoma characterized by abnormalities in PDGFRA, IDH1, EGFR, and NF1. Cancer Cell. 2010;17(1):98-110.

4. Furnari FB, Fenton T, Bachoo RM, Mukasa A, Stommel JM, Stegh A, et al. Malignant astrocytic glioma: genetics, biology, and paths to treatment. Genes Dev. 2007;21(21):2683-710.

5. Ohgaki H, Kleihues P. The definition of primary and secondary glioblastoma. Clin Cancer Res. 2013;19(4):764-72.

6. Consortium EP. An integrated encyclopedia of DNA elements in the human genome. Nature. 2012;489(7414):57-74.

7. Rinn JL, Chang HY. Genome regulation by long noncoding RNAs. Annu Rev Biochem. 2012;81:145-66.

8. Peng Z, Liu C, Wu M. New insights into long noncoding RNAs and their roles in glioma. Mol Cancer. 2018;17(1):61.

9. Yoon JH, Kim J, Gorospe M. Long noncoding RNA turnover. Biochimie. 2015;117:15-21.

10. Yang WB, Hsu CC, Hsu TI, Liou JP, Chang KY, Chen PY, et al. Increased activation of $\mathrm{HDAC} 1 / 2 / 6$ and SP1 underlies therapeutic resistance and tumor growth in glioblastoma. Neuro-Oncology. 2020;22(10):1439-51.

11. Lee HY, Tsai AC, Chen MC, Shen PJ, Cheng YC, Kuo CC, et al. Azaindolylsulfonamides, with a more selective inhibitory effect on histone deacetylase 6 activity, exhibit antitumor activity in colorectal cancer HCT116 cells. J Med Chem. 2014;57(10):4009-22.

12. Li T, Zhang C, Hassan S, Liu X, Song F, Chen K, et al. Histone deacetylase 6 in cancer. J Hematol Oncol. 2018;11(1):111.

13. Deguchi S, Katsushima K, Hatanaka A, Shinjo K, Ohka F, Wakabayashi $T$, et al. Oncogenic effects of evolutionarily conserved noncoding RNA ECONEXIN on gliomagenesis. Oncogene. 2017;36(32):4629-40.

14. Deng M, Yuan H, Liu S, Hu Z, Xiao H. Exosome-transmitted LINC00461 promotes multiple myeloma cell proliferation and suppresses apoptosis by modulating microRNA/BCL-2 expression. Cytotherapy 2019;21(1):96-106.

15. Dong L, Qian J, Chen F, Fan Y, Long J. LINC00461 promotes cell migration and invasion in breast cancer through miR-30a-5p/integrin beta3 axis. J Cell Biochem. 2019;120(4):4851-62.
16. Yang WB, Chuang JY, Ko CY, Chang WC, Hsu TI. Dehydroepiandrosterone induces temozolomide resistance through modulating phosphorylation and acetylation of Sp1 in glioblastoma. Mol Neurobiol. 2019;56(4):2301-13.

17. Jensen MM, Jorgensen JT, Binderup T, Kjaer A. Tumor volume in subcutaneous mouse xenografts measured by microCT is more accurate and reproducible than determined by $18 \mathrm{~F}-F D G-m i c r o P E T$ or external caliper. BMC Med Imaging. 2008;8:16.

18. Chang KY, Hsu TI, Hsu CC, Tsai SY, Liu JJ, Chou SW, et al. Specificity protein 1-modulated superoxide dismutase 2 enhances temozolomide resistance in glioblastoma, which is independent of O(6)-methylguanine-DNA methyltransferase. Redox Biol. 2017;13:655-64.

19. Crowe AR, Yue W. Semi-quantitative determination of protein expression using immunohistochemistry staining and analysis: an integrated protocol. Bio Protoc. 2019;9(24):e3465.

20. Goswami CP, Nakshatri H. PROGgeneV2: enhancements on the existing database. BMC Cancer. 2014;14:970.

21. Chang KY, Huang CT, Hsu TI, Hsu CC, Liu JJ, Chuang CK, et al. Stress stimuli induce cancer-stemness gene expression via Spl activation leading to therapeutic resistance in glioblastoma. Biochem Biophys Res Commun. 2017;493(1):14-9.

22. Sharma S, Poetz F, Bruer M, Ly-Hartig TB, Schott J, Seraphin B, et al. Acetylation-dependent control of global poly(A) RNA degradation by CBP/p300 and HDAC1/2. Mol Cell. 2016;63(6):927-38.

23. Udagawa T, Fujioka Y, Tanaka M, Honda D, Yokoi S, Riku Y, et al. FUS regulates AMPA receptor function and FTLD/ALS-associated behaviour via GluA1 mRNA stabilization. Nat Commun. 2015;6:7098.

24. Chen $Y$, Wang $X$. miRDB: an online database for prediction of functional microRNA targets. Nucleic Acids Res. 2020;48(D1):D127-D31.

25. Dweep H, Gretz N. miRWalk2.0: a comprehensive atlas of microRNAtarget interactions. Nat Methods. 2015;12(8):697.

26. Li JH, Liu S, Zhou H, Qu LH, Yang JH. starBase v2.0: decoding miRNAceRNA, miRNA-ncRNA and protein-RNA interaction networks from large-scale CLIP-Seq data. Nucleic Acids Res. 2014;42(Database issue):D92-7.

27. Zhang Y, Sui R, Chen Y, Liang H, Shi J, Piao H. Downregulation of miR485-3p promotes glioblastoma cell proliferation and migration via targeting RNF135. Exp Ther Med. 2019;18(1):475-82.

28. Statello L, Guo CJ, Chen LL, Huarte M. Gene regulation by long non-coding RNAs and its biological functions. Nat Rev Mol Cell Biol. 2021;22(2):96-118.

29. Schmitt AM, Chang HY. Long noncoding RNAs in cancer pathways. Cancer Cell. 2016;29(4):452-63.

30. Hsu CC, Chang WC, Hsu TI, Liu JJ, Yeh SH, Wang JY, et al. Suberoylanilide hydroxamic acid represses glioma stem-like cells. J Biomed Sci. 2016:23(1):81.

31. Ji D, Wang Y, Li H, Sun B, Luo X. Long non-coding RNA LINC00461/miR149-5p/LRIG2 axis regulates hepatocellular carcinoma progression. Biochem Biophys Res Commun. 2019;512(2):176-81.

32. Qu W, Huang W, Yang F, Ju H, Zhu G. Long noncoding RNA LINC0046 mediates cisplatin resistance of rectal cancer via miR-593-5p/CCND1 axis. Biomed Pharmacother. 2020;124:109740.

33. Guttman M, Amit I, Garber M, French C, Lin MF, Feldser D, et al. Chromatin signature reveals over a thousand highly conserved large non-coding RNAs in mammals. Nature. 2009;458(7235):223-7.

34. Wu X, Brewer $G$. The regulation of mRNA stability in mammalian cells: 2.0. Gene. 2012;500(1):10-21.

35. Statello L, Guo CJ, Chen LL, Huarte M. Author correction: Gene regulation by long non-coding RNAs and its biological functions. Nat Rev Mol Cell Biol. 2021;22(2):159.

36. Bartlam M, Yamamoto T. The structural basis for deadenylation by the CCR4-NOT complex. Protein Cell. 2010;1(5):443-52.

37. Arenas A, Chen J, Kuang L, Barnett KR, Kasarskis EJ, Gal J, et al. Lysine acetylation regulates the RNA binding, subcellular localization and inclusion formation of FUS. Hum Mol Genet. 2020;29(16):2684-97.

38. Pommier Y, Leo E, Zhang H, Marchand C. DNA topoisomerases and their poisoning by anticancer and antibacterial drugs. Chem Biol. 2010;17(5):421-33.

39. Yang Y, Ren M, Song C, Li D, Soomro SH, Xiong Y, et al. LINC00461, a long non-coding RNA, is important for the proliferation and migration of glioma cells. Oncotarget. 2017;8(48):84123-39. 
40. Hwang B, Lee JH, Bang D. Single-cell RNA sequencing technologies and bioinformatics pipelines. Exp Mol Med. 2018;50(8):1-14.

41. Carlevaro-Fita J, Johnson R. Global positioning system: understanding Long noncoding RNAs through subcellular localization. Mol Cell. 2019;73(5):869-83.

42. Noh JH, Kim KM, McClusky WG, Abdelmohsen K, Gorospe M. Cytoplasmic functions of long noncoding RNAs. Wiley Interdiscip Rev RNA. 2018;9(3):e1471.

\section{Publisher's Note}

Springer Nature remains neutral with regard to jurisdictional claims in published maps and institutional affiliations.

- fast, convenient online submission

- thorough peer review by experienced researchers in your field

- rapid publication on acceptance

- support for research data, including large and complex data types

- gold Open Access which fosters wider collaboration and increased citations

- maximum visibility for your research: over 100M website views per year

At BMC, research is always in progress.

Learn more biomedcentral.com/submissions 